NEIP-05-02

hep-th/0502225

February/October 2005

\title{
Five-Brane Thresholds and Membrane Instantons in Four-Dimensional Heterotic M-Theory
}

\author{
Luca Carlevaro $^{1}$ and Jean-Pierre Derendinger ${ }^{2}$ \\ Physics Institute, Neuchâtel University, \\ A.-L. Breguet 1, CH-2000 Neuchâtel, Switzerland
}

\begin{abstract}
The effective four-dimensional supergravity of M-theory compactified on the orbifold $S^{1} / Z_{2}$ and a Calabi-Yau threefold includes in general moduli supermultiplets describing massless modes of five-branes. For each brane, one of these fields corresponds to fluctuations along the interval. The fivebrane also leads to modifications of the anomaly-cancelling terms in the eleven-dimensional theory, including gauge contributions located on their world-volumes. We obtain the interactions of the brane "interval modulus" predicted by these five-brane-induced anomaly-cancelling terms and we construct their effective supergravity description. In the condensed phase, these interaction terms generate an effective non-perturbative superpotential which can also be interpreted as instanton effects of open membranes stretching between five-branes and the $S^{1} / Z_{2}$ fixed hyperplanes. Aspects of the vacuum structure of the effective supergravity are also briefly discussed.
\end{abstract}

1 luca.carlevaro@unine.ch

2 jean-pierre.derendinger@unine.ch 


\section{Introduction}

Heterotic $E_{8} \times E_{8}$ strings compactified to four dimensions on a six-dimensional space $K_{6}$ are also described by M-theory compactified on $K_{7} \equiv S^{1} / Z_{2} \times K_{6}$ [1, 2]. In particular, it is straightforward to verify [3, 4, 5, 6] that the effective $\mathcal{N}_{4}=1$ supergravity found in Calabi-Yau or orbifold compactifications of perturbative heterotic strings [7, 8] is reproduced by brane-free M-theory configurations with compact space $K_{7}$. A novelty of the M-theory approach lies in the possibility to concretely analyse physical effects of non-perturbative brane configurations. In the low-energy effective supergravity approximation, configurations with five-branes and/or membranes (two-branes) 9, 10, 1] of compactified M-theory can be studied from simple modifications of the field equations predicted by eleven-dimensional supergravity [11].

An obvious distinction in the nature of five-brane and membrane effects follows from the alignement conditions applying to their respective world-volumes if one requires that the configuration admits (exact or spontaneously broken) $\mathcal{N}_{4}=1$ supersymmetry (four supercharges). Each five-brane world-volume is the product of four-dimensional space-time and a holomorphic two-cycle in the Calabi-Yau threefold and conditions apply on the respective cycles of pairs of world-volumes [10, 1]. Five-brane massless excitations [12, which belong to six-dimensional chiral supersymmetry multiplets expanded in modes of the two-cycle, lead then to new four-dimensional fields to be included in the effective supergravity description. Some of these modes do not depend on the detail of the Calabi-Yau geometry: the five-brane modulus describing fluctuations along the $S^{1} / Z_{2}$ direction, the two-index antisymmetric tensor $\hat{B}_{\mu \nu}$ with self-dual field strength and their fermionic $\mathcal{N}_{4}=1$ partner. These states can be assembled either in a chiral supermultiplet which we will call $\hat{S}$ or, in a dual version, in a linear multiplet. The effective supergravity for this "universal five-brane modulus" supermultiplet has been studied in ref. [13] (see also ref. [14]) ${ }^{1}$. Firstly, the Kähler potential of the theory with this new superfield has been obtained and the absence of direct contributions to the (perturbative) superpotential has been demonstrated. Secondly, on the basis of the four-dimensional superspace structure only, the possible appearance of new threshold corrections has been emphasized.

In contrast, open membrane euclidean world-volumes include the $S^{1} / Z_{2}$ direction and a cycle in $K_{6}$ [10, 17]. They stretch between the $S^{1} / Z_{2}$ fixed planes, or between a fixed plane and a five-brane, or between pairs of five-branes. Their effects in the fourdimensional effective supergravity are then localized in space-time, they can be viewed as instanton-like corrections to the interaction Lagrangian. While open membrane

\footnotetext{
${ }^{1}$ And, as a function of a non-trivial background value of the five-brane modulus, ref. [15, 16].
} 
stretching between the fixed hyperplanes correspond in the string approach to worldsheet instantons, membranes ending on a five-brane describe forces acting on this brane. Their contributions to the effective supergravity are then expected to lead to new (nonderivative since the world-volume includes $S^{1} / Z_{2}$ ) interactions involving the five-brane modulus.

The corrections to the effective four-dimensional supergravity induced by the various types of membranes have been studied in refs. [14, 18, 19]. They were found to contribute to the chiral $F$-density part of the Lagrangian density, in the form of a non-perturbative superpotential. Specifically, an interaction bilinear in the five-brane fermion in superfield $\hat{S}$ has been computed in the four-dimensional background with the five-brane and open membranes ending on it. The resulting non-perturbative superpotential shows an exponential dependence on the five-brane universal modulus typical of instanton calculus. To isolate the membrane contributions from other possible non-perturbative sources, a specific regime is chosen. ${ }^{2}$ As a consequence, even if the instanton calculation clearly establishes the existence of an exponential dependence on $\hat{S}$, it does not allow to infer how this exponential term would combine with other non-perturbative contributions which, like gauge instantons, are expected as well. ${ }^{3}$

The relevance to physics of the M-theory system with five-branes and membranes strongly relies upon the structure of superpotentials generated by fluxes, gaugino condensates and open membrane instantons. Phenomenological questions addressed in the literature include supersymmetry breaking and gaugino condensation [16, 19], fivebrane stabilization (stabilization of the modulus $\hat{S}$ ) [14, 19], stabilization of all moduli [20], the existence of stable de Sitter vacua [20, 21, inflationaly phases and potentials [21] and cosmic strings [22]. These analyses use in general simplifying assumptions, in the Kähler metric which shows a severe mixing of all moduli when five-brane fields are present, or in the superpotential which is assumed to be a simple sum of nonperturbative contributions.

In the present paper, we use the anomaly-cancelling terms of the eleven-dimensional theory on the orbifold $S^{1} / Z_{2}$, as modified when five-branes are present, to derive new interactions involving the five-brane universal modulus supermultiplet which describes fluctuations along the $S^{1} / Z_{2}$ orbifold direction. These new interactions are then shown to induce, in the condensed phase, the effective non-perturbative instanton superpotential expected from membranes stretching between a fixed hyperplane and a five-brane. This superpotential correctly reduces to the results of refs. [14, 18, 19] in the regime

\footnotetext{
${ }^{2}$ For instance, Moore, Peradze and Saulina 14 select a regime where "open membrane instanton effects are the leading source of non-perturbative effects".

${ }^{3}$ Writing the complete non-perturbative superpotential as a sum of contributions, as for instance in ref. [14, is an assumption which needs to be justified.
} 
considered in these articles, but its derivation does not require choosing a particular limiting regime. This approach provides then direct information on the non-perturbative superpotential with combined gauge and membrane instantons effects.

The fact that these four-dimensional interactions can be obtained by considering seemingly unrelated arguments (membrane instanton calculus or gauge anomalycancelling terms) is a consequence of the superfield structure of the four-dimensional theory. We use an effective superfield formulation [23, 13] which fully respects the symmetry and supersymmetry ingredients defining the microscopic system: the modifications of the Bianchi identities and of the topological term induced by fixed planes and five-branes. It also respects the symmetries of the five-brane multiplet, with its self-dual three-form field. Thus, a given superspace contribution in the effective Lagrangian describes various aspects of the microscopic theory, related by the superfield structure of the effective theory. This method has been applied to the derivation of the Kähler potential [13], including non-linear couplings of the five-brane superfield $\hat{S}$, and we will see in paragraph 4.1 that these kinetic terms can be derived from (at least) two quite distinct sectors of the microscopic theory. They can certainly be derived from the Calabi-Yau reduction of the five-brane Born-Infeld Lagrangian [13]. But they can also be derived from a universal correction to gauge kinetic terms, quadratic in $\hat{S}$, induced by $S^{1} / Z_{2}$ anomaly-cancellation.

Schematically, our argument goes as follows. Since we confine ourselves to the effective four-dimensional supergravity with up to two derivatives, for which a (superconformal) superspace formulation exists, counterterms cancelling Lorentz anomalies will be irrelevant to our discussion. ${ }^{4}$ Gauge anomaly-cancelling terms are then entirely due to the "topological term" of eleven-dimensional supergravity [11]

$$
-\frac{1}{24 \kappa^{2}} \int C_{3} \wedge G_{4} \wedge G_{4}
$$

Sources for the Bianchi identity verified by $G_{4}$ are provided by the two fixed hyperplanes of the $S^{1} / Z_{2}$ orbifold and by the aligned five-branes, so that

$$
G_{4}=d C_{3}+\Delta G_{4, \text { planes }}+\Delta G_{4, \text { branes }}
$$

The contribution $\Delta G_{4, \text { planes }}$ depends on the gauge curvatures living on the planes, and both corrections explicitly depend on the $S^{1} / Z_{2}$ coordinate and respect the $Z_{2}$ symmetry used to define the orbifold projection. The topological term leads then to a gauge interaction of the form

$$
-\frac{1}{12 \kappa^{2}} \int C_{3} \wedge \Delta G_{4, \text { planes }} \wedge \Delta G_{4, \text { branes }}
$$

\footnotetext{
${ }^{4}$ They would however lead to similar phenomena.
} 
This term gives rise in particular to a gauge interaction of the massless orbifold modes of $C_{3}$ located on the five-brane world-volumes and depending explicitly on their position along $S^{1}$. And, after integration over the Calabi-Yau space, it produces a coupling to $F \wedge F$ of the axionic partner $\operatorname{Im} T$ of the Calabi-Yau volume modulus ${ }^{5} \operatorname{Re} T$ which depends on the five-brane locations along $S^{1}$. The superfield structure developed for the effective supergravity of the universal five-brane modulus [13] can then be used to understand this interaction as a threshold correction with a calculable dependence on the five-brane modulus. In particular, the universal part of these contributions can be derived from the Dirac-Born-Infeld kinetic Lagrangian. The non-perturbative effective superpotential follows then from standard gaugino condensation. Its dependence on the five-brane modulus is precisely the one expected from membrane instanton calculations, as performed for instance in ref. [14].

Along similar lines, a description of some new charged matter contributions arising from M-theory anomaly-cancellation can be given. An interesting feature is that the structure organizing five-brane threshold corrections is carried over to these matter interactions.

The paper is organised as follows. The anomaly counterterm at the origin of the five-brane-related gauge interactions is derived in Section [2. The reduction to four dimensions and the identification of the obtained terms as superfield densities are then discussed in Sections 3 and 4. Then, in Section 5. condensation is performed to derive the non-perturbative superpotential, compare it with membrane instanton results and discuss some physical consequences. Conclusions and comments are added in Section 6] and an appendix collects conventions, notations and technical details.

\section{The anomaly counterterm}

Ten-dimensional hyperplanes located at the $Z_{2}$ fixed points along $S^{1}$ and five-branes act as sources of the Bianchi identity verified by the four-form field $G_{4}$ of elevendimensional supergravity. As a consequence of these contributions, the topological term acquires anomalous variations under local symmetries. Together with the variations of the Green-Schwarz term [24, 1, 2, 9], of order four in the Riemann curvature, these anomalous variations are precisely those required to cancel perturbative gauge and Lorentz anomalies generated by the $Z_{2}$ orbifold projection of the eleven-dimensional

\footnotetext{
${ }^{5}$ For the bulk moduli $T$ and $S$, we use the terminology familiar from string compactifications in which $T$ is the volume modulus and $S$ the dilaton or string coupling modulus. The terminology commonly used in the context of $M$-theory, as for instance in refs. 14, 15, is unfortunately different. Our conventions are precisely stated in the appendix.
} 
theory and by the chiral gauge multiplets living on the hyperplanes.

The modification of the Bianchi identity and of its solution $G_{4}$ of course leads to modifications of the effective action. All modifications generated by the topological term would have more than two derivatives in the heterotic, ten-dimensional, small $S^{1}$ radius limit. But compactifying further to four dimensions on the Calabi-Yau space $K_{6}$ also generates modifications of the effective action at the level of two-derivative gauge terms, because of the non-trivial background values of $\left\langle\operatorname{tr} R^{2}\right\rangle$ and $\left\langle\operatorname{tr} F^{2}\right\rangle$. The purpose of this section is to precisely derive some of these terms which arise whenever five-branes are present. We then begin by recalling some aspects of the description of $M$-theory on the orbifold $S^{1} / Z_{2}$. It should be noted that some ambiguities remain in our understanding of this description. The gauge sector relevant to our problem escapes however these ambiguities.

The explicit formulation of the modified Bianchi identity uses two types of sources, associated with hyperplanes supporting Yang-Mills ten-dimensional supermultiplets and five branes world-volumes. On both hyperplanes (labelled by $i=1,2$ ) live gauge supermultiplets with curvature two-forms $F_{i}$. The quantity appearing in the Bianchi identity is the gauge invariant four-form

$$
I_{4, i}=\frac{1}{(4 \pi)^{2}}\left[\frac{1}{30} \operatorname{Tr} F_{i}^{2}-\frac{1}{2} \operatorname{tr} R^{2}\right], \quad d I_{4, i}=0, \quad(i=1,2)
$$

where $R$ is the Lorentz curvature two-form. Five-branes compatible with $\mathcal{N}_{4}=1$ (four supercharges) supersymmetry wrap space-time $M_{4}$ and a two-cycle in $K_{6}$. The transverse Dirac distribution $\delta_{\mathcal{W}_{6, I}}^{(5)}$ for five-brane number $I$ with world-volume $\mathcal{W}_{6, I}$ is the five-brane source in the Bianchi identity, which then reads ${ }^{6}$

$$
d G_{4}=2\left(4 \pi \kappa^{2}\right)^{1 / 3}\left[\frac{1}{2} \sum_{I} \delta^{(5)}\left(\mathcal{W}_{6, I}\right)-\sum_{i} \delta_{i} I_{4, i}\right]
$$

The one-forms

$$
\delta_{1}=\delta(y) d y, \quad \delta_{2}=\delta(y-\pi) d y
$$

localize the gauge sources on the $Z_{2}$-fixed hyperplanes. ${ }^{7}$ In order to respect the $Z_{2}$ symmetry used in the orbifold projection, we actually label with index $I$ pairs of fivebranes located at $\pm y_{I} .^{8}$

The procedure to resolve ambiguities in the solution of the Bianchi identity has been discussed in detail in the literature. ${ }^{9}$ The general solution of eq. (2.2) includes several

\footnotetext{
${ }^{6}$ Supersymmetry forbids that both five-branes and anti-five-branes couple to the $S^{1} / Z_{2}$ orbifold.

${ }^{7}$ The appendix collects our conventions.

${ }^{8}$ And one may then choose $0 \leq y_{I} \leq \pi$.

${ }^{9}$ For instance in refs. [25] and [26], and references therein.
} 
arbitrary integration constants which are constrained by consistency conditions: the four-form field $G_{4}$ should be gauge-invariant and globally well-defined, its action should be well-defined, the fields eliminated by the $Z_{2}$ truncation should be gauge-invariant, as well as the massive modes of the $S^{1}$ expansion of the surviving states. These conditions should be verified for any number of five-branes at arbitrary locations. Writing

$$
G_{4}=d C_{3}+\Delta G_{4, \text { branes }}+\Delta G_{4, \text { planes }}
$$

the consistency constraints point to a unique solution for the hyperplane contribution ${ }^{10}$ :

$$
\begin{aligned}
\Delta G_{4, \text { planes }} & =-\left(4 \pi \kappa^{2}\right)^{1 / 3} \sum_{i}\left(\epsilon_{i} I_{4, i}-\frac{d y}{\pi} \wedge \omega_{3, i}\right), \\
& =-\left(4 \pi \kappa^{2}\right)^{1 / 3} \sum_{i}\left(d\left[\epsilon_{i} \omega_{3, i}\right]-2 \delta_{i} \wedge \omega_{3, i}\right),
\end{aligned}
$$

where, as discussed in detail in the appendix,

$$
\epsilon_{i}(y)=\operatorname{sgn}\left(y-y_{i}\right)-\frac{y-y_{i}}{\pi}, \quad y_{1}=0, \quad y_{2}=\pi, \quad y \in[-\pi, \pi]
$$

and the Chern-Simons three-forms are defined by

$$
d \omega_{3, i}=I_{4, i}, \quad i=1,2 .
$$

A similar discussion can be made for the five-brane contribution to $G_{4}$. As already mentioned, the five-branes are space-time filling and wrap a holomorphic two-cycle in $K_{6}$. This implies that one can certainly write

$$
\delta^{(5)}\left(\mathcal{W}_{6, I}\right)=\left[\delta\left(y-y_{I}\right)+\delta\left(y+y_{I}\right)\right] d y \wedge \delta^{(4)}\left(\mathcal{W}_{6, I}\right)
$$

where $\delta^{(4)}\left(\mathcal{W}_{6, I}\right)$ is now a four-form Dirac distribution in $K_{6}$ such that

$$
\int_{M_{4} \times K_{6}} I_{6} \wedge \delta^{(4)}\left(\mathcal{W}_{6, I}\right)=\int_{\mathcal{W}_{6, I}} I_{6}
$$

for any six-form $I_{6}$. The natural solution of the Bianchi identity is then to include in $G_{4}$ the brane contribution

$$
\begin{aligned}
\Delta G_{4, \text { branes }}= & \left(4 \pi \kappa^{2}\right)^{1 / 3} \sum_{I}\left[\frac{1}{2} \hat{\epsilon}_{y_{I}}(y) \delta^{(4)}\left(\mathcal{W}_{6, I}\right)-\frac{1}{\pi} d y \wedge \theta^{(3)}\left(\mathcal{W}_{6, I}\right)\right]+d \mathcal{C}_{3} \\
= & -\left(4 \pi \kappa^{2}\right)^{1 / 3} \sum_{I}\left\{\delta\left(y-y_{I}\right)+\delta\left(y+y_{I}\right)\right\} d y \wedge \theta^{(3)}\left(\mathcal{W}_{6, I}\right) \\
& +d\left\{\mathcal{C}_{3}+\frac{1}{2}\left(4 \pi \kappa^{2}\right)^{1 / 3} \sum_{I} \hat{\epsilon}_{y_{I}}(y) \theta^{(3)}\left(\mathcal{W}_{6, I}\right)\right\}
\end{aligned}
$$

where $d \theta^{(3)}\left(\mathcal{W}_{6, I}\right)=\delta^{(4)}\left(\mathcal{W}_{6, I}\right)$ and

$$
\begin{aligned}
& \hat{\epsilon}_{y_{I}}(y)=\operatorname{sgn}\left(y-y_{I}\right)+\operatorname{sgn}\left(y+y_{I}\right)-\frac{2 y}{\pi}, \quad 0 \leq y_{I} \leq \pi, \\
& d \hat{\epsilon}_{y_{I}}(y)=2\left[\delta\left(y-y_{I}\right)+\delta\left(y+y_{I}\right)-\frac{1}{\pi}\right] d y .
\end{aligned}
$$

\footnotetext{
${ }^{10}$ This is the solution with " $b=1$ " in refs. [25, 26].
} 
Notice that $\epsilon_{1}(y)=\frac{1}{2} \hat{\epsilon}_{0}(y)$ and $\epsilon_{2}(y)=\frac{1}{2} \hat{\epsilon}_{\pi}(y)$. The addition in eq. (2.9) of the term $d \mathcal{C}_{3}$ allows for the introduction of brane modes contributions into the topological term, if necessary [26]: it is known [27, 28] that a gauging by $C_{3}$ of the three-form field of fivebrane fluctuations is induced by consistent coupling of five-branes to eleven-dimensional supergravity. We will not elaborate on this point here since in four dimensions the needed terms would automatically appear in the supersymmetrization of the effective theory. ${ }^{11}$

To summarize, one can write

$$
\begin{aligned}
G_{4}= & d \tilde{C}_{3}+2\left(4 \pi \kappa^{2}\right)^{1 / 3} \sum_{i} \delta_{i} \wedge \omega_{3, i} \\
& -\left(4 \pi \kappa^{2}\right)^{1 / 3} \sum_{I}\left\{\delta\left(y-y_{I}\right)+\delta\left(y+y_{I}\right)\right\} d y \wedge \theta^{(3)}\left(\mathcal{W}_{6, I}\right), \\
\tilde{C}_{3}= & C_{3}-\left(4 \pi \kappa^{2}\right)^{1 / 3}\left[\sum_{i} \epsilon_{i} \omega_{3, i}-\frac{1}{2} \sum_{I} \hat{\epsilon}_{y_{I}}(y) \theta^{(3)}\left(\mathcal{W}_{6, I}\right)\right]+\mathcal{C}_{3},
\end{aligned}
$$

and $G_{4}$ and $d \tilde{C}_{3}$ only differ at locations where hyperplanes or five-branes sit.

The gauge transformation of $C_{3}$ is completely fixed by consistency of the $Z_{2}$ orbifold projection. The topological term indicates that $C_{3}$ is intrinsically odd. The components $C_{A B C}, A, B, C=0 \ldots, 9$, are then projected out and should then for consistency be gauge invariant. This condition implies

$$
\begin{aligned}
\delta C_{3} & =\left(4 \pi \kappa^{2}\right)^{1 / 3} \frac{1}{\pi} d y \wedge \sum_{i} \omega_{2, i}^{1} \\
\delta \tilde{C}_{3} & =\left(4 \pi \kappa^{2}\right)^{1 / 3} \sum_{i}\left[2 \delta_{i} \wedge \omega_{2, i}^{1}-d\left\{\epsilon_{i} \omega_{2, i}^{1}\right\}\right]
\end{aligned}
$$

where $\delta \omega_{3, i}=d \omega_{2, i}^{1}$. The correct modified topological term, which cancels anomalies locally, is then [26]

$$
-\frac{1}{24 \kappa^{2}} \int \tilde{C}_{3} \wedge G_{4} \wedge G_{4}
$$

Our goal is to infer from this modified topological term four-dimensional interactions of the massless $S^{1} / Z_{2} \times K_{6}$ modes. The substitution of eqs. (2.11) leads to two classes of terms. Firstly, contributions involving the massless mode $C_{A B y}(A, B=0, \ldots, 9)$. This mode corresponds to the antisymmetric tensor $B_{A B}$ of ten-dimensional sixteensupercharge supergravity. From gauge-transformation (2.12), one deduces that the appropriate definition with $\delta B=(4 \pi)^{2} \sum_{i} \omega_{2, i}^{1}$ is ${ }^{12}$

$$
B_{A B}=\left(4 \pi \kappa^{2}\right)^{-1 / 3}(4 \pi)^{2} \pi R_{S^{1}} C_{A B y}^{(0)}, \quad C_{A B y}^{(0)}=\frac{1}{2 \pi} \int_{-\pi}^{\pi} d y C_{A B y}
$$

\footnotetext{
${ }^{11}$ See Section 4

${ }^{12}$ Taking into account the factor $(4 \pi)^{-2}$ in the definitions of $I_{4, i}$ and $\omega_{3, i}$. This is the definition of the two-form field commonly used in ten dimensions, with dimension (mass) ${ }^{2}$.
} 
where $R_{S^{1}}$ is the $S^{1}$ radius. In terms of this massless field, the topological term produces the following four-dimensional interactions

$$
-\frac{1}{32 \pi^{2}} \int_{S^{1} \times K_{6}} B_{2} \wedge\left[\sum_{i, j} \epsilon_{i} \epsilon_{j} I_{4, i} \wedge I_{4, j}-\sum_{i, I} \epsilon_{i} \hat{\epsilon}_{y_{I}} I_{4, i} \wedge \delta^{(4)}\left(\mathcal{W}_{6, I}\right)\right]
$$

with $\int_{S^{1}}=\int_{-\pi}^{\pi} d y$. The first contribution, when integrated over $S^{1}$ only, generates the Green-Schwarz gauge anomaly-cancelling terms expected for the $E_{8} \times E_{8}$ heterotic string 24]. Its consequences for Calabi-Yau compactifications have been studied long ago [29, 8]. When integrated over $S^{1} \times K_{6}$, it leads to gauge threshold corrections depending on the Calabi-Yau volume modulus as well as dilaton-dependent chargedmatter terms. The second contribution, which also depends on the $S^{1}$ position of the five-branes, is of interest to us.

The modified topological term also produces the following $C_{3}$-independent gauge interactions:

$$
\begin{aligned}
-\frac{1}{3} \int_{S^{1} \times K_{6}} & {\left[-\sum_{i, j, k} \epsilon_{i} \epsilon_{j} \omega_{3, i} \wedge I_{4, j} \wedge \omega_{3, k}+\frac{1}{2} \sum_{i, J, k} \epsilon_{i} \hat{\epsilon}_{y_{J}} \omega_{3, i} \wedge \delta^{(4)}\left(\mathcal{W}_{6, J}\right) \wedge \omega_{3, k}\right.} \\
+ & \left.\frac{1}{2} \sum_{i, J, k} \epsilon_{i} \hat{\epsilon}_{y_{J}} I_{4, i} \wedge \theta^{(3)}\left(\mathcal{W}_{6, J}\right) \wedge \omega_{3, k}+\sum_{i, j, K} \epsilon_{i} \epsilon_{j} \omega_{3, i} \wedge I_{4, j} \wedge \theta^{(3)}\left(\mathcal{W}_{6, K}\right)\right] .
\end{aligned}
$$

After integration over $y$, the first contribution is a local counterterm allowed by anomaly cancellation [26] ${ }^{13}$. The next three terms are non-trivial brane contributions depending on their positions along $S^{1}$.

The overlap integrals over $S^{1}$ give an interesting result. First introduce the numbers

$$
d_{i j}=\int_{-\pi}^{\pi} d y \epsilon_{i} \epsilon_{j}=\frac{\pi}{3}\left(3 \delta_{i j}-1\right)
$$

for the first integrals in contributions (2.15) and (2.16). For those depending on the brane position, define the variables

$$
\Delta_{I, 1}=\frac{y_{I}}{\pi}, \quad \Delta_{I, 2}=1-\frac{y_{I}}{\pi}, \quad\left(0<y_{I}<\pi ; \quad 0<\Delta_{I, j}<1\right),
$$

the distances from brane $I$ to the fixed planes, with normalization $\Delta_{I, 1}+\Delta_{I, 2}=1$. Then,

$$
\begin{aligned}
& \frac{1}{2 \pi} \int_{-\pi}^{\pi} d y \epsilon_{1}(y) \hat{\epsilon}_{y_{I}}(y)=\left(\Delta_{I, 2}\right)^{2}-\frac{1}{3}=\frac{y_{I}^{2}}{\pi^{2}}-2 \frac{y_{I}}{\pi}+\frac{2}{3}, \\
& \frac{1}{2 \pi} \int_{-\pi}^{\pi} d y \epsilon_{2}(y) \hat{\epsilon}_{y_{I}}(y)=\left(\Delta_{I, 1}\right)^{2}-\frac{1}{3}=\frac{y_{I}^{2}}{\pi^{2}}-\frac{1}{3} .
\end{aligned}
$$

\footnotetext{
${ }^{13}$ The anomaly twelve-form obtained from descent equations vanishes.
} 
It will be important for the supersymmetrization of the four-dimensional interactions that the terms quadratic in $y_{I}$ are identical in both integrals. With these results, contributions (2.15) lead to

$$
\begin{aligned}
& -\frac{1}{16 \pi} \int_{K_{6}} B_{2} \wedge\left[\frac{1}{3}\left(I_{4,1}^{2}+I_{4,2}^{2}-I_{4,1} I_{4,2}\right)\right. \\
& \left.-\sum_{I} \delta^{(4)}\left(\mathcal{W}_{6, I}\right) \wedge\left\{\left(\Delta_{I, 2}^{2}-\frac{1}{3}\right) I_{4,1}+\left(\Delta_{I, 1}^{2}-\frac{1}{3}\right) I_{4,2}\right\}\right],
\end{aligned}
$$

while expressions (2.16) give

$$
\begin{aligned}
\frac{\pi}{3} \int_{K_{6}}\left\{\omega_{3,1} \wedge \omega_{3,2} \wedge\right. & \left(I_{4,1}-I_{4,2}\right)+\frac{3}{2} \sum_{I}\left(\Delta_{I, 1}-\Delta_{I, 2}\right) \delta^{(4)}\left(\mathcal{W}_{6, I}\right) \wedge \omega_{3,1} \wedge \omega_{3,2} \\
-\sum_{I} \theta^{(3)}\left(\mathcal{W}_{6, I}\right) \wedge & {\left[\left(\Delta_{I, 2} \omega_{3,1}-\Delta_{I, 1} \omega_{3,2}\right) \wedge\left(\Delta_{I, 2} I_{4,1}-\Delta_{I, 1} I_{4,2}\right)\right.} \\
-\omega_{3,1} & \left.\left.\wedge I_{4,1}-\omega_{3,2} \wedge I_{4,2}+\frac{1}{2} \omega_{3,1} \wedge I_{4,2}+\frac{1}{2} \omega_{3,2} \wedge I_{4,1}\right]\right\}
\end{aligned}
$$

after some partial integrations.

The anomalous gauge variation of contributions (2.20) and (2.21) can be written as

$$
\begin{aligned}
& -\frac{\pi}{3}\left(\omega_{2,1}^{1} I_{4,1}^{2}+\omega_{2,2}^{1} I_{4,2}^{2}\right) \\
& +\frac{2 \pi}{3} \sum_{I} \delta^{(4)}\left(\mathcal{W}_{6, I}\right) \wedge\left[\Delta_{I, 2} \omega_{2,1}^{1}-\Delta_{I, 1} \omega_{2,2}^{1}\right] \wedge\left[\Delta_{I, 2} I_{4,1}-\Delta_{I, 1} I_{4,2}\right] .
\end{aligned}
$$

Applying descent equations to these variations leads to the formal anomaly twelve-form

$$
\begin{aligned}
\mathcal{I}_{12}= & -\frac{\pi}{3}\left[I_{4,1}+I_{4,2}-\sum_{I} \delta^{(4)}\left(\mathcal{W}_{6, I}\right)\right] \\
& \wedge\left[I_{4,1}^{2}+I_{4,2}^{2}-I_{4,1} I_{4,2}+\sum_{I} \delta^{(4)}\left(\mathcal{W}_{6, I}\right) \wedge\left\{\left(1-3 \Delta_{I, 2}^{2}\right) I_{4,1}+\left(1-3 \Delta_{I, 1}^{2}\right) I_{4,2}\right\}\right] \\
= & \mathcal{I}_{12, \text { het. }}+\sum_{I} \delta^{(4)}\left(\mathcal{W}_{6, I}\right) \mathcal{I}_{8, I},
\end{aligned}
$$

since for four-dimensional space-time-filling five-branes, $\delta^{(4)}\left(\mathcal{W}_{6, I}\right) \wedge \delta^{(4)}\left(\mathcal{W}_{6, J}\right)=0$. The contribution of each five-brane is encoded in the eight-form

$$
\mathcal{I}_{8, I}=\pi\left[\Delta_{I, 2} I_{4,1}-\Delta_{I, 1} I_{4,2}\right]^{2}
$$

while the heterotic contribution is as usual $\mathcal{I}_{12, \text { het. }}=-\frac{\pi}{3}\left[I_{4,1}^{3}+I_{4,2}^{3}\right]$.

The form of $\mathcal{I}_{8, I}$, eq. (2.24), clearly shows that the distance from the brane to the first plane acts as the (inverse squared) coupling of the gauge fields living on the second 
plane. Similarly, the distance from the brane to the second plane acts as the (inverse squared) coupling of the gauge fields living on the first plane.

At this point, the conclusion is that the effective, four-dimensional supergravity includes gauge contributions due to five-branes which arise from the Calabi-Yau reduction of expressions (2.20) and (2.21), as derived from the modified topological term (2.13). In the next two sections, we perform this reduction keeping only the "universal" massless modes unrelated to geometrical details of $K_{6}$, but including the five-brane modulus along the $S^{1}$ direction, and we write the effective four-dimensional supergravity using superconformal tensor calculus.

\section{Reduction to four dimensions}

In the reduction to four dimensions, we use the freedom to rescale moduli fields to set the $S^{1}$ circumference $2 \pi R_{S^{1}}$ and the Calabi-Yau volume $V_{6}$ to unity. ${ }^{14}$

As usual, the massless modes of the metric tensor expanded on $M_{4} \times K_{7}$ include $g_{\mu \nu}$, the scalar field $g_{y y}$ and massless modes of the internal metric $g_{i \bar{k}}$. Among these, we only keep the universal, Kähler-metric volume modulus. Similarly, the antisymmetric tensor $C_{A B y}$ leads to a massless $B_{\mu \nu}$ and we only keep the universal massless mode of the internal tensor $B_{i \bar{k}}$. With these bosonic modes and their fermionic partners, the reduction of eleven-dimensional supergravity can be described by two chiral multiplets $S$ and $T$ with the familiar Kähler potential [7, [8] ${ }^{15}$

$$
\mathcal{K}=-\ln (S+\bar{S})-3 \ln (T+\bar{T}) .
$$

Following eq. (2.14), we define

$$
B_{\mu \nu}=\frac{(4 \pi)^{2}}{2}\left(4 \pi \kappa^{2}\right)^{-1 / 3} C_{\mu \nu y}, \quad B_{i \bar{j}}=\frac{i}{\kappa_{4}^{2}} \operatorname{Im} T \delta_{i \bar{j}}
$$

and $B_{\mu \nu}$ is dual to $\operatorname{Im} S$.

With five-branes, vector fields on the two fixed hyperplanes gauge an algebra $G_{1} \times G_{2}$ which is further broken by the Calabi-Yau compactification. Embedding the $S U(3)$ holonomy into $G_{1} \times G_{2}$ defines the four-dimensional gauge group $G_{(4)}$ as the stabilizer of this $S U(3)$ in $G_{1} \times G_{2}$. Calabi-Yau reduction of the ten-dimensional gauge fields ${ }^{16}$

\footnotetext{
${ }^{14}$ The four-dimensional gravitational constant is then $\kappa_{4}^{2}=\kappa^{2}$, but we nevertheless use different symbols since their mass dimensions differ.

${ }^{15}$ In general, we use the same notation for a chiral supermultiplet and for its lowest complex scalar component.

${ }^{16}$ We find useful to keep track of the plane index $i=1,2$ and $\alpha(i)$ is then an index in the adjoint representation of $G_{i}$. Similarly, $a(i)$ will be used for the adjoint of $G_{(4)}$ and $m(i)$ for the representation spanned by complex scalar fields.
} 
$\mathcal{A}_{B}^{\alpha(i)}$ leads then to the corresponding gauge fields $\mathcal{A}_{\mu}^{a(i)}$. It also produces a set of $S U(3)$-singlet complex scalar fields $\mathcal{A}^{m(i)}$ in some representation of $G_{(4)}$.

With up to two derivatives, Riemann curvature contributions in counterterms (2.20) 2.21) can be omitted. The Calabi-Yau reduction of $I_{4, i}$ delivers then:

$$
\begin{aligned}
I_{i, \mu \nu \rho \sigma} & =\frac{3 !}{(4 \pi)^{2}} \sum_{\alpha} F_{[\mu \nu}^{\alpha(i)} F_{\rho \sigma]}^{\alpha(i)} \\
I_{i, \mu \nu k \bar{l}} & =-\frac{4}{(4 \pi)^{2}} \sum_{m}\left(\mathcal{D}_{[\mu} \mathcal{A}^{m(i)}\right)\left(\mathcal{D}_{\nu]} \overline{\mathcal{A}}^{m(i)}\right) \delta_{k \bar{l}} \\
& =\frac{2 i}{(4 \pi)^{2}} \sum_{m} \partial_{[\mu}\left(\mathcal{A}^{m(i)} \mathcal{D}_{\nu]} \overline{\mathcal{A}}^{m(i)}-\overline{\mathcal{A}}^{m(i)} \mathcal{D}_{\nu]} \mathcal{A}^{m(i)}\right) i \delta_{k \bar{l}} \\
I_{i, \mu j k l} & =\frac{2}{(4 \pi)^{2}} \partial_{\mu}\left(\lambda_{i, m n p} \mathcal{A}^{m(i)} \mathcal{A}^{n(i)} \mathcal{A}^{p(i)}\right) \epsilon_{j k l}
\end{aligned}
$$

In the last expression, $\lambda_{i, m n p}$ is the symmetric tensor invariant under $G_{(4)}$ that may arise from the internal Chern-Simons term $\omega_{i, j k l}$. We will use the notations

$$
\lambda \mathcal{A}^{3}=\sum_{i} \lambda \mathcal{A}_{i}^{3}, \quad \lambda \mathcal{A}_{i}^{3} \equiv \lambda_{i, m n p} \mathcal{A}^{m(i)} \mathcal{A}^{n(i)} \mathcal{A}^{p(i)}
$$

to denote this cubic holomorphic couplings which also appear in the superpotential

$$
W=c+\lambda \mathcal{A}^{3}
$$

Finally, $I_{4, i}$ has a non-trivial background value $\left\langle I_{4, i}\right\rangle_{i j \overline{k l}}$.

With these results, the reduction to four dimensions of the first line in expression (2.20), which depends on $B_{2}$ and exists without five-brane can be written

$$
\begin{aligned}
\Delta \mathcal{L}_{\text {plane }}= & \frac{1}{2(4 \pi)^{4}} \sum_{i, j} d_{i j}\left\{\frac{1}{\kappa_{4}^{2}} e_{4}\left\langle\mathcal{I}_{j}\right\rangle \operatorname{Im} T F_{\mu \nu}^{\alpha(i)} \widetilde{F}^{\alpha(i) \mu \nu}\right. \\
& -i\left\langle\mathcal{I}_{j}\right\rangle \epsilon^{\mu \nu \rho \sigma}\left(\partial_{\mu} B_{\nu \rho}\right) \sum_{m}\left[\mathcal{A}^{m(i)}\left(\mathcal{D}_{\sigma} \overline{\mathcal{A}}^{m(i)}\right)-\overline{\mathcal{A}}^{m(i)}\left(\mathcal{D}_{\sigma} \mathcal{A}^{m(i)}\right)\right] \\
& \left.-\frac{i}{(4 \pi)^{2}} \epsilon^{\mu \nu \rho \sigma}\left(\partial_{\mu} B_{\nu \rho}\right)\left(\lambda \mathcal{A}_{i}^{3} \partial_{\sigma}\left(\overline{\lambda \mathcal{A}}_{j}^{3}\right)-\partial_{\sigma}\left(\lambda \mathcal{A}_{i}^{3}\right) \overline{\lambda \mathcal{A}}_{j}^{3}\right)\right\}
\end{aligned}
$$

The background value of $I_{4, i}$ is encoded in the integral over the Calabi-Yau manifold

$$
\left\langle\mathcal{I}_{i}\right\rangle=V_{6}^{-1} \int_{K_{6}}\left\langle I_{i}\right\rangle_{k l k l} \delta_{j \bar{j}} \epsilon^{j k l} \epsilon^{\overline{j k l}} .
$$

In expression (3.6), the first term is a threshold correction depending on the volume modulus already well-known from the heterotic strings [8, 29]. The second and third 
contributions describe interactions of matter scalars with the string coupling multiplet. They have been considered in detail in refs. [3, 5, 23].

The reduction of the five-brane contribution in expression (2.20) leads to the following Lagrangian terms:

$$
\begin{aligned}
\Delta \mathcal{L}_{\text {brane }}=\frac{1}{8(4 \pi)^{3}} \sum_{I, i} a_{I}\left(\Delta_{I, i}^{2}-\frac{1}{3}\right)\left[\frac{1}{\kappa_{4}^{2}} e_{4} \operatorname{Im} T F_{\mu \nu}^{\alpha(i)} \widetilde{F}^{\alpha(i) \mu \nu}\right. \\
\left.\quad-i \epsilon^{\mu \nu \rho \sigma} \partial_{\mu} B_{\nu \rho} \sum_{m}\left[\mathcal{A}^{m(i)}\left(\mathcal{D}_{\sigma} \overline{\mathcal{A}}^{m(i)}\right)-\overline{\mathcal{A}}^{m(i)}\left(\mathcal{D}_{\sigma} \mathcal{A}^{m(i)}\right)\right]\right] .
\end{aligned}
$$

The area of the Calabi-Yau two-cycle (with coordinate $z$ ) wrapped by the five-brane world-volume can be written

$$
a_{I}=\int_{\mathcal{C}_{2, I}} d z d \bar{z} \frac{\partial z^{m}}{\partial z} \frac{\partial \bar{z}^{\bar{n}}}{\partial \bar{z}} \delta_{m \bar{n}} .
$$

The first term in (3.8) is the five-brane contribution to gauge threshold corrections. Its existence has been demonstrated in an explicit background calculation by Lukas, Ovrut and Waldram [15, 16]. The second term is again a matter interaction with the string coupling multiplet. Both terms depend on the positions $y_{I}$ of the five-branes. Hence, they depend on the $S^{1} / Z_{2}$ five-brane modulus.

The terms collected in expression (2.21) are somewhat ambiguous since they are defined up to contributions which, like the first one or any counterterm of the form $\theta^{(3)}\left(\mathcal{W}_{6, I}\right) \wedge I_{7}$, do not contribute to the gauge-invariant anomaly twelve-form. To reduce the first term, introduce the four-dimensional Chern-Simons forms

$$
\begin{array}{ll}
\partial_{[\mu} \omega_{i, \nu \rho \sigma]}=\frac{1}{4} F_{i,[\mu \nu} F_{i, \rho \sigma]}, & \partial_{[\mu} \omega_{i, \nu] j \bar{k}}=\frac{1}{2} I_{i, \mu \nu j \bar{k}}, \\
\partial_{\mu} \omega_{i, j k l}=I_{i, \mu j k l} . &
\end{array}
$$

The first term then generates couplings of charged matter scalars to gauge fields:

$$
\begin{aligned}
\Delta \mathcal{L}_{\text {plane }}= & \frac{i}{3(4 \pi)^{5}} \epsilon^{\mu \nu \rho \sigma} \omega_{1, \mu \nu \rho}\left[\lambda \mathcal{A}_{2}^{3} \partial_{\sigma}\left(\overline{\lambda \mathcal{A}}_{2}^{3}\right)-\overline{\lambda \mathcal{A}}_{2}^{3} \partial_{\sigma}\left(\lambda \mathcal{A}_{2}^{3}\right)+\lambda \mathcal{A}_{1}^{3} \partial_{\sigma}\left(\overline{\lambda \mathcal{A}}_{2}^{3}\right)\right. \\
& \left.-\overline{\lambda \mathcal{A}}_{1}^{3} \partial_{\sigma}\left(\lambda \mathcal{A}_{2}^{3}\right)+2\left[\bar{\lambda}_{2}^{3} \partial_{\sigma}\left(\lambda \mathcal{A}_{1}^{3}\right)-\lambda \mathcal{A}_{2}^{3} \partial_{\sigma}\left(\overline{\lambda \mathcal{A}}_{1}^{3}\right)\right]\right]+(1 \leftrightarrow 2) \\
& -\frac{i}{12(4 \pi)^{3}} \epsilon^{\mu \nu \rho \sigma} \omega_{1, \mu \nu \rho} \sum_{m}\left(\mathcal{A}^{m(2)} \mathcal{D}_{\sigma} \overline{\mathcal{A}}^{m(2)}-\overline{\mathcal{A}}^{m(2)} \mathcal{D}_{\sigma} \mathcal{A}^{m(2)}\right)\left(\left\langle\mathcal{I}_{1}\right\rangle-\left\langle\mathcal{I}_{2}\right\rangle\right) \\
& +(1 \leftrightarrow 2) .
\end{aligned}
$$

As we will see in the next section, these terms do not have a natural supersymmetric extension in general, a fact which may have some relation to their ambiguous character. 
Notice however that in the minimal embedding of the Calabi-Yau background into one plane only, most of these mixing terms are absent and a natural supersymmetrization exists.

Likewise, the second term in eq. (2.21) yields gauge--matter interactions depending on the five-brane positions along $S^{1}$ :

$$
\begin{aligned}
\Delta \mathcal{L}_{\text {brane }}=\frac{i}{8(4 \pi)^{3}} \sum_{I} a_{I}\left(\Delta_{I, 1}-\Delta_{I, 2}\right) \epsilon^{\mu \nu \rho \sigma} \omega_{1, \mu \nu \rho} & \\
& \times \sum_{m}\left[\mathcal{A}^{m(2)}\left(\mathcal{D}_{\sigma} \overline{\mathcal{A}}^{m(2)}\right)-\overline{\mathcal{A}}^{m(2)}\left(\mathcal{D}_{\sigma} \mathcal{A}^{m(2)}\right)\right]+(1 \leftrightarrow 2) .
\end{aligned}
$$

In the next section, we will derive the effective four-dimensional supergravity in the particular case of a single five-brane. To simplify, we will omit the index $I$ and the corresponding sums. We will however use a formulation in which restoring contributions of several branes is straightforward.

\section{The effective supergravity}

The universal $S^{1} / Z_{2}$ five-brane modulus describing fluctuations along the interval direction has a supersymmetric bosonic partner arising from the mode expansion of the five-brane antisymmetric tensor $\hat{B}_{m n}$. Six-dimensional (world-volume) supersymmetry of the five-brane multiplet requires that the three-form curvature $\hat{H}_{m n p}$ of this tensor is self-dual. For the massless four-dimensional universal mode, self-duality is the condition $^{17}$

$$
\hat{H}_{\mu \nu \rho} \equiv 3 \partial_{[\mu} \hat{B}_{\nu \rho]}=e_{4} \epsilon_{\mu \nu \rho}{ }^{\sigma} \partial_{\sigma} \hat{B}_{i \bar{j}} \equiv e_{4} \epsilon_{\mu \nu \rho}{ }^{\sigma} \hat{H}_{\sigma i \bar{j}}
$$

Then, clearly, the four-dimensional supersymmetric description of the five-brane universal modulus uses either a linear multiplet $\hat{L}$ with the tensor $\hat{H}_{\mu \nu \rho}$ and a real scalar $\hat{C}$ for the modulus, or a chiral multiplet with complex scalar $\hat{S}$ and $\operatorname{Im} \hat{S}$ related to $\hat{B}_{i \bar{j}}$. The supersymmetric extension of condition (4.1) is chiral-linear duality, the duality transformation exchanging superfields $\hat{S}$ and $\hat{L}$ [30].

The fact that the chiral multiplet $\hat{S}$ is dual to a linear multiplet has three important consequences for its supergravity couplings. Firstly, the Kähler potential is a function of $\hat{S}+\bar{S}$ only. Secondly, the holomorphic gauge kinetic function can only depend linearly on $\hat{S}$. These two consequences follow from the intrinsic gauge invariance of $\hat{B}_{\mu \nu}$, which translates into axionic shift symmetry of $\hat{S}$ in the chiral formulation. Thirdly,

\footnotetext{
${ }^{17}$ Omitting fermion and covariantization contributions.
} 
the superpotential does not depend on $\hat{S}$. In supergravity, in contrast to global supersymmetry, this statement is ambiguous and directly linked to the first consequence above. The superpotential $W$ and the Kähler potential $\mathcal{K}$ are not independent: the entire theory depends only on $\mathcal{G}=\mathcal{K}+\ln |W|^{2}$. Terms can then be moved from or into $\mathcal{K}$ provided they are harmonic functions of the complex chiral fields. The correct statement is then that $\mathcal{G}$ may only depend on $\hat{S}+\overline{\hat{S}}$. Moving terms from $\mathcal{K}$ to the superpotential can artificially generate a dependence on $\hat{S}$ of the form

$$
W_{\text {new }}=e^{b \hat{S}} W\left(z^{i}\right)
$$

where $b$ is a real constant and $z^{i}$ denotes all other chiral multiplets, and a new Kähler potential $\mathcal{K}_{\text {new }}\left(\hat{S}+\overline{\hat{S}}, z^{i}, \bar{z}_{i}\right)$ such that the function $\mathcal{G}$ remains unchanged, $\mathcal{K}+\ln |W|^{2}=\mathcal{K}_{\text {new }}+\ln \left|W_{\text {new }}\right|^{2}$. Notice that adding a $\hat{S}$-independent term to the superpotential (4.2) is not allowed. Non-perturbative exponential superpotentials generated, for instance, by gaugino condensation, and added to a perturbative superpotential are then incompatible with chiral-linear duality. ${ }^{18}$

The effective four-dimensional supergravity depends on three moduli multiplets coupled to supergravity, gauge and charged matter superfields. Each of the three moduli scalars has a component of an antisymmetric tensor as supersymmetry partner. More precisely, the string coupling modulus is in the multiplet describing $G_{\mu \nu \rho y}$, the $K_{6}$ volume modulus is paired with $G_{\mu i \bar{k} y}$ and the five-brane $S^{1} / Z_{2}$ modulus is the partner of the components $\hat{H}_{\mu \nu \rho}$ or $\hat{H}_{\mu i \bar{k}}$ of the self-dual antisymmetric tensor living on the brane world-volume. We find, as explained in ref. [23, more efficient to formulate the theory using superconformal tensor calculus and to introduce three moduli vector superfields to describe these moduli multiplets: ${ }^{19}$

$$
\begin{array}{lll}
V & (w=2, n=0): & G_{\mu \nu \rho y}, \text { string coupling modulus, } \ldots \\
V_{T} & (w=n=0): & G_{\mu i \bar{k} y}, \text { Calabi-Yau volume modulus, } \ldots \\
\hat{V} & (w=n=0): & \hat{H}_{\mu \nu \rho}, \text { five-brane } S^{1} / Z_{2} \text { modulus, ... }
\end{array}
$$

The components of the antisymmetric tensors are identified with the vector fields in these multiplets, the moduli scalars with their real lowest components. These vector multiplets are then submitted to Bianchi identities obtained from the $K_{7}$ reduction of the eleven-dimensional Bianchi identity for $G_{4}$, eq. (2.2), and the self-duality condition of the five-brane tensor. In each case, the supersymmetrized Bianchi identity also reduces the number of off-shell states from $8_{B}+8_{F}$ in a vector multiplet to $4_{B}+4_{F}$. These Bianchi identities are imposed as the field equations of three Lagrange-multiplier superfields:

\footnotetext{
${ }^{18}$ See however ref. 31 for an analysis.

${ }^{19}$ The Weyl weight is $w$ and $n$ is the chiral weight.
} 
$S \quad(w=n=0): \quad$ a chiral multiplet for the Bianchi identity verified by $V$,

$L_{T} \quad(w=2, n=0): \quad$ a linear multiplet for the Bianchi identity verified by $V_{T}$,

$\hat{S} \quad(w=n=0): \quad$ a chiral multiplet for the self-duality condition of the brane tensor, applied to $\hat{V}$.

Eliminating these Lagrange multiplier superfields defines the three vector multiplets in terms of the physical fields solving Bianchi identities. The important advantage of this procedure is that supersymmetrizing the theory before eliminating Lagrange multipliers automatically delivers the correct non-linear couplings of source terms (brane and plane contributions) to moduli and then the correct Kähler potential. Alternatively, equivalent (dual) versions of the theory can be obtained by eliminating some vector multiplets instead of the Lagrange multipliers.

These six multiplets describing bulk and brane states are supplemented by states living on the fixed hyperplanes. In the notation defined in the previous section, these states include gauge chiral superfields $\mathcal{W}^{\alpha(i)}(i=1,2$ as usual, $w=n=3 / 2)$ and charged matter chiral multiplets $(w=n=0)$ in some representation of the gauge group. They will be collectively denoted by $M$ and they contain the complex scalar components $\mathcal{A}^{m(i)}$. Finally, we need the compensating chiral multiplet $S_{0}(w=n=1)$ to gauge fix the superconformal theory to super-Poincaré symmetry only.

With this set of superfields, the Lagrangian nicely splits in a sum of five terms with well-defined higher-dimensional interpretations:

$$
\mathcal{L}=\mathcal{L}_{\text {bulk }}+\mathcal{L}_{\text {Bianchi }}+\mathcal{L}_{\text {kinetic }}+\mathcal{L}_{\text {thresholds }}+\mathcal{L}_{\text {superpotential }}
$$

The bulk Lagrangian 32

$$
\mathcal{L}_{\text {bulk }}=-\left[\left(S_{0} \bar{S}_{0} V_{T}\right)^{3 / 2}(2 V)^{-1 / 2}\right]_{D}
$$

can be directly obtained from the $K_{7}$ reduction of eleven-dimensional supergravity, expressed in terms of $G_{4}$. It depends on $V$ (string coupling multiplet) and $V_{T}\left(K_{6}\right.$ volume modulus multiplet), and of the compensator $S_{0}$. In eq. (4.4), $[\ldots]_{D}$ denotes the invariant real density formula of superconformal calculus, as reviewed and developed in for instance refs. 33]. Similarly, $[\ldots]_{F}$ will below denote the chiral density formula. ${ }^{20}$

The coupling of plane and brane fields $\left(\mathcal{W}^{\alpha(i)}, M\right.$ and $\left.\hat{V}\right)$ to bulk multiplets is entirely encoded in $\mathcal{L}_{\text {Bianchi }}$, which reads [13]

$$
\mathcal{L}_{\text {Bianch } i}=\left[-(S+\bar{S})\left(V+2 \Omega_{1}+2 \Omega_{2}\right)+L_{T}\left(V_{T}+2 \bar{M} e^{2 A} M\right)+\frac{1}{2} \tau(\hat{S}+\overline{\hat{S}}) V \hat{V}\right]_{D},
$$

where $\Omega_{1}$ and $\Omega_{2}$ are the Chern-Simons multiplets $(w=2, n=0)$ for the gauge algebra arising on each hyperplane, defined by ${ }^{21} \sum_{\alpha} \mathcal{W}^{\alpha(i)} \mathcal{W}^{\alpha(i)}=16 \Sigma\left(\Omega_{i}\right)$, and $\bar{M} e^{2 A} M \equiv$

\footnotetext{
${ }^{20}$ In global supersymmetry, $[\ldots]_{D}$ and $[\ldots]_{F}$ would be $\int d^{4} \theta[\ldots]$ and $\int d^{2} \theta[\ldots]+$ h.c.

${ }^{21}$ The operation $\Sigma(\ldots)$ is the superconformal analog of $\frac{1}{8} \overline{\mathcal{D D}}$ in global superspace.
} 
$\sum_{m, i} \bar{M}^{m(i)}\left[e^{2 A} M\right]^{m(i)}$ is the Wess-Zumino Lagrangian superfield. This contribution is gauge-invariant since $\left[(S+\bar{S})\left(\delta \Omega_{1}+\delta \Omega_{2}\right)\right]_{D}$ is a derivative. The dimensionless number $\tau$ is proportional to the five-brane tension $T_{5}$. In our units, it reads

$$
\tau=\frac{2}{(4 \pi)^{3}} a,
$$

where $a$ is the area of the two-cycle wrapped by the brane in $K_{6}$, as defined in eq. (3.9). Notice shift symmetries $\delta S=i c, \delta \hat{S}=i d(c, d$ real).

The kinetic terms of the five-brane fields arise from reduction of the Dirac-BornInfeld (DBI) Lagrangian:

$$
\mathcal{L}_{\text {kinetic }}=-\tau\left[V V_{T} \hat{V}^{2}\right]_{D}
$$

They are quadratic in $\hat{V}$, a consequence of the form of the DBI action, and the prefactor $V V_{T}$ is the coupling to the supergravity background. Notice that since this term is linear in $V$, it will naturally assemble with the contribution in $S+\bar{S}$ in eq. (4.5).

At this point, the contributions from bulk, plane and five-brane kinetic Lagrangians have been considered, with tensor fields in $V, V_{T}$ and $\hat{V}$ verifying Bianchi identities modified by plane and brane sources. But we still have to consider further contributions from the topological term with modified $G_{4}$, as obtained in the previous section. These terms will be collected in $\mathcal{L}_{\text {threshold }}$. The symmetries of expressions (4.4)-(4.7) allow the introduction of the following corrections to gauge kinetic terms [13] (threshold corrections):

$$
\begin{aligned}
\mathcal{L}_{\text {thresholds }}= & {\left[-2 \sum_{i} \beta_{i} \Omega_{i}\left(V_{T}+2 \bar{M} e^{2 A} M\right)\right]_{D} } \\
& +\frac{\tau}{4}\left[\hat{S} \sum_{i} \hat{\beta}_{i} \mathcal{W}_{i} \mathcal{W}_{i}\right]_{F}+\left[V\left\{\epsilon\left|\alpha M^{3}\right|^{2}-2 \tau \sum_{i} g_{i}(\hat{V}) \bar{M}^{i} e^{2 A} M^{i}\right\}\right]_{D} .
\end{aligned}
$$

The first contribution corresponds to threshold corrections depending on the volume modulus [8, 29, 34]. Gauge invariance of the full Lagrangian with this term is obtained by postulating the appropriate variation of the linear multiplet $L_{T}$ in $\mathcal{L}_{\text {Bianchi }}$ :

$$
\delta L_{T}=2 \sum_{i} \beta_{i} \delta \Omega_{i} .
$$

The second and third contributions are threshold corrections depending on the $S^{1} / Z_{2}$ location of the five-brane and/or, for the last one, on matter multiplets. The coefficients $\beta_{i}, \hat{\beta}_{i}, \epsilon$ and the functions $g_{i}(\hat{V})$ can be obtained, as explained below, from Calabi-Yau reduction of the topological term with anomaly-cancelling modifications. Symmetries of the theory leave these functions unconstrained but the terms considered here only require linear functions, $g_{i}(\hat{V})=\hat{\gamma}_{i} \hat{V}+\hat{\delta}_{i}$. Finally, the quantity $\alpha M^{3}$ in expression (4.8) denotes the holomorphic cubic invariant also present in the matter superpotential [7, 8]. 
The superpotential arises from the components $G_{i j k y}$ of $G_{4}$. They also verify a nontrivial Bianchi identity which is not modified by five-branes since three holomorphic directions cannot be transverse to their world-volumes. The superpotential contribution to the Lagrangian is then

$$
\mathcal{L}_{\text {superpotential }}=\left[S_{0}^{3} W\right]_{F}, \quad \text { with } \quad W=c+\alpha M^{3},
$$

The constant $c$ being the 'flux' of the heterotic three-form in direction $\epsilon_{i j k}$ [7, 35].

This formulation of the effective supergravity, with six superfields to describe three moduli supermultiplets leads to several equivalent forms, depending on the choice made when eliminating the three superfluous multiplets. The Lagrange multipliers $S, L_{T}$ and $\hat{S}$ imply the following constraints on the vector multiplets:

$$
\begin{array}{rlrlrl}
S: & V & =L-2 \sum_{i} \Omega_{i}, & & L \text { linear }(w=2, n=0), \\
L_{T}: & V_{T} & =T+\bar{T}-2 \bar{M} e^{2 A} M, & & T \text { chiral }(w=n=0), \\
\hat{S}: & \hat{V} & =V^{-1}\left(\hat{L}+4 \sum_{i} \hat{\beta}_{i} \Omega_{i}\right), & \hat{L} \text { linear }(w=2, n=0) .
\end{array}
$$

Eliminating $S, L_{T}$ and $\hat{S}$ leads then to a formulation where moduli are described by $L, T$ and $\hat{L}$, two linear and one chiral multiplets:

$$
\begin{aligned}
\mathcal{L}= & {\left[-\frac{1}{\sqrt{2}}\left[S_{0} \bar{S}_{0}\left(T+\bar{T}-2 \bar{M} e^{2 A} M\right)\right]^{3 / 2}\left(L-2 \sum_{i} \Omega_{i}\right)^{-1 / 2}\right.} \\
& -\tau\left(T+\bar{T}-2 \bar{M} e^{2 A} M\right)\left(L-2 \sum_{i} \Omega_{i}\right)^{-1}\left(\hat{L}+4 \sum_{j} \hat{\beta}_{j} \Omega_{j}\right)^{2} \\
& +\left(L-2 \sum_{i} \Omega_{i}\right)\left\{\epsilon\left|\alpha M^{3}\right|^{2}-2 \tau \sum_{j} \hat{\delta}_{j} \bar{M}^{j} e^{2 A} M^{j}\right\} \\
& \left.-2 \tau\left(\hat{L}+4 \sum_{i} \hat{\beta}_{i} \Omega_{i}\right) \sum_{j} \hat{\gamma}_{j} \bar{M}^{j} e^{2 A} M^{j}\right]_{D} \\
& +\left[S_{0}^{3} W+\frac{1}{4} \sum_{i} \beta_{i} T \mathcal{W}_{i} \mathcal{W}_{i}\right]_{F} .
\end{aligned}
$$

Component expressions for this apparently complicated theory can be obtained using superconformal tensor calculus [33]. Notice that plane contributions (superfields $\Omega_{i}, \mathcal{W}_{i}$ and $M^{i}$ ) now appear in the bulk Lagrangian (first line) and also in the five-brane DBI contribution (second line). Five-brane contributions (multiplet $\hat{L}$ ) appear in gauge kinetic terms (a "plane term") while threshold corrections (third and fourth lines) involve plane and five-brane fields. These mixings are induced by the modified Bianchi identities, eqs. (4.10), and by threshold corrections required by anomaly cancellation.

The kinetic term quadratic in the five-brane modulus superfield $\hat{L}$ appears in the second line. It can clearly be derived from the DBI Lagrangian, as done in ref. [13]. But the superfield structure implied by the modified Bianchi identity leading to the first eq. 
(4.10) also implies that the same kinetic term can be obtained from gauge threshold corrections which follow from Calabi-Yau reduction of the (modified) topological term. This point will be explicitly verified in the next paragraph.

This unfamiliar supergravity theory is particularly useful to study its scalar potential and vacuum structure since linear multiplets do not have auxiliary fields. We will come back to this point later on and especially when studying condensation.

It is however more common to formulate the supergravity theory with chiral moduli $S$ and $T$, and then to eliminate $V$ and $L_{T}$ using their field equations. One obtains:

$$
\mathcal{L}=-\frac{3}{2}\left[S_{0} \bar{S}_{0} e^{-\mathcal{K} / 3}\right]_{D}+\left[S_{0}^{3} W+\frac{1}{4} \sum_{i}\left(S+\beta_{i} T+\tau \hat{\beta}_{i} \hat{S}\right) \mathcal{W}^{i} \mathcal{W}^{i}\right]_{F},
$$

with the Kähler potential

$$
\mathcal{K}=-\ln (S+\bar{S}-\Delta)-3 \ln \left(T+\bar{T}-2 \bar{M} e^{2 A} M\right)
$$

and

$$
\Delta=-\tau\left(T+\bar{T}-2 \bar{M} e^{2 A} M\right) \hat{V}^{2}+\frac{1}{2} \tau(\hat{S}+\overline{\hat{S}}) \hat{V}+\epsilon\left|\alpha M^{3}\right|^{2}-2 \tau \sum_{i} g_{i}(\hat{V}) \bar{M}^{i} e^{2 A} M^{i}
$$

The field equation of the vector multiplet $\hat{V}$ implies then

$$
\hat{V}=\left(4 V_{T}\right)^{-1}\left(\hat{S}+\overline{\hat{S}}-4 \sum_{i} \hat{\gamma}_{i} \bar{M}^{i} e^{2 A} M^{i}\right)
$$

with $V_{T}$ as in the second eq. (4.10). The fully chiral formulation of the effective supergravity is then defined by Kähler potential (4.13) with now

$$
\begin{aligned}
\Delta= & \frac{\tau}{16}\left(T+\bar{T}-2 \bar{M} e^{2 A} M\right)^{-1}\left(\hat{S}+\overline{\hat{S}}-4 \sum_{i} \hat{\gamma}_{i} \bar{M}^{i} e^{2 A} M^{i}\right)^{2} \\
& +\epsilon\left|\alpha M^{3}\right|^{2}-2 \tau \sum_{i} \hat{\delta}_{i} \bar{M}^{i} e^{2 A} M^{i}
\end{aligned}
$$

gauge kinetic functions

$$
f^{i}=S+\beta^{i} T+\tau \hat{\beta}^{i} \hat{S}
$$

and superpotential (4.9). The presence of the five-brane then introduces mixing of the three chiral multiplets $S, T$ and $\hat{S}$ and the off-diagonal elements of the Kähler metric severely complicate the analysis of the theory.

Before returning to the analysis of the effective supergravity, we need a precise identification of the supergravity fields in terms of massless modes of the $K_{7}$ compactification.

The notation we use for component fields is as follows. Vector multiplets $V, \hat{V}$ and $V_{T}$ have respectively vector fields $V_{\mu}, \hat{V}_{\mu}$ and $T_{\mu}$ and (lowest) real scalar $C, \hat{C}$ and $C_{T}$. 
And we use the same notation for chiral multiplets $S, T$ and $\hat{S}$ and for their complex scalar lowest components. Eqs. (4.10) indicate then that

$$
C_{T}=2(\operatorname{Re} T-\bar{M} M), \quad T_{\mu}=-2 \partial_{\mu} \operatorname{Im} T-2 i \bar{M}\left(\mathcal{D}_{\mu} M\right)+2 i\left(\mathcal{D}_{\mu} \bar{M}\right) M
$$

that the lowest scalar component of the string coupling linear multiplet $L$ is also $C$ and that the five-brane linear multiplet $\hat{L}$ has a real scalar $\hat{\ell}=C \hat{C}$. Relation (4.15) also implies that

$$
\begin{aligned}
\operatorname{Re} \hat{S}= & 4 \hat{C}(\operatorname{Re} T-\bar{M} M)+2 \sum_{i} \hat{\gamma}_{i} \bar{M}^{i} M^{i} \\
\partial_{\mu} \operatorname{Im} \hat{S}= & -4 \hat{V}_{\mu}(\operatorname{Re} T-\bar{M} M)+4 \hat{C}\left(\partial_{\mu} \operatorname{Im} T+i \bar{M} \mathcal{D}_{\mu} M-i M \mathcal{D}_{\mu} \bar{M}\right) \\
& -2 i \sum_{i} \hat{\gamma}_{i}\left(\bar{M}^{i} \mathcal{D}_{\mu} M^{i}-M^{i} \mathcal{D}_{\mu} \bar{M}^{i}\right)
\end{aligned}
$$

The scalar field $\hat{C}$ has background value proportional to $y$, the five-brane location along $S^{1}$. In our units with $2 \pi R_{S^{1}}=1=V_{6}$, the four-dimensional gravitational constant is $\kappa_{4}=\kappa$ and

$$
\langle\hat{C}\rangle=\frac{1}{\kappa_{4}} y R_{S^{1}}=\frac{y}{2 \pi \kappa}
$$

In order to derive the identification of the matter scalars $\mathcal{A}^{m(i)}$ of eq. (3.3) and the superconformal multiplets $M^{m(i)}$, we note that the component $G_{\mu i \bar{j} y}$ of the four-form is related to the vector component of the real multiplet $V_{T}$, which is

$$
\left(V_{T}\right)_{\mu}=-2\left[\partial_{\mu} \operatorname{Im} T+i \bar{M}\left(\mathcal{D}_{\mu} M\right)-i\left(\mathcal{D}_{\mu} \bar{M}\right) M\right]
$$

On the other hand, using eqs. (3.2) and (3.3), we also find

$$
\begin{aligned}
G_{\mu i \bar{j} y} & =\partial_{\mu} C_{i \bar{j} y}-\frac{\left(4 \pi \kappa^{2}\right)^{1 / 3}}{\pi} \sum_{k} \omega_{k, \mu i \bar{j}} \\
& =\frac{1}{2 \pi}\left(4 \pi \kappa^{2}\right)^{-2 / 3}\left(\partial_{\mu} \operatorname{Im} T-i \frac{\kappa^{2}}{2 \pi}\left[\mathcal{A}^{(i)} \mathcal{D}_{\mu} \overline{\mathcal{A}}^{(i)}-\overline{\mathcal{A}}^{(i)} \mathcal{D}_{\mu} \mathcal{A}^{(i)}\right]\right) \delta_{i \bar{j}}
\end{aligned}
$$

By comparison, one obtains

$$
\mathcal{A}^{(i)}=\frac{\sqrt{2 \pi}}{\kappa} M^{(i)}
$$

with an irrelevant sign choice.

Finally, the gauge fields in $\Omega_{i}$ or $\mathcal{W}^{a(i)}$ are the massless modes $\mathcal{A}_{\mu}^{a(i)}$.

\subsection{Gauge coupling constants}

The effective supergravity Lagrangian predicts a very specific moduli dependence in the super-Yang-Mills sector relevant to the determination of the effective superpotential with non-perturbative configurations. The field-dependent gauge couplings can of 
course be obtained in any formulation of the theory. But the closest relation to the higher-dimensional origin of the effective supergravity is realized with supermultiplets $L$ (for the string dilaton multiplet), $T$ (Calabi-Yau volume modulus) and $\hat{L}$ (for the five-brane $S^{1} / Z_{2}$ modulus). This is the theory defined by eq. (4.11) in which tensor calculus leads to the following (inverse squared) gauge couplings:

$$
\begin{aligned}
\frac{1}{g_{i}^{2}}= & \frac{1}{2}\left(\frac{z_{0} \bar{z}_{0}(T+\bar{T}-2 \bar{M} M)}{2 C}\right)^{3 / 2}+\frac{\tau}{2}(T+\bar{T}-2 \bar{M} M)\left[\hat{C}^{2}+4 \hat{\beta}_{i} \hat{C}\right] \\
& +\beta_{i} \operatorname{Re} T+\frac{1}{2}\left[\epsilon\left|\alpha M^{3}\right|^{2}-2 \tau \sum_{j}\left(\hat{\delta}_{j}-\hat{\beta}_{i} \hat{\gamma}_{j}\right) \bar{M}^{j} M^{j}\right]
\end{aligned}
$$

The complex scalar $z_{0}$ is the lowest component of the compensating multiplet $S_{0}$. In the Poincaré theory, it is a function of the physical scalars chosen to obtain a specific "gravity frame". The Einstein frame where the gravity Lagrangian is $-\frac{1}{2 \kappa_{4}^{2}} e_{4} R_{4}$ corresponds to ${ }^{22}$

$$
2 \kappa_{4}^{2} C=\left(\frac{z_{0} \bar{z}_{0}(T+\bar{T}-2 \bar{M} M)}{2 C}\right)^{-3 / 2} .
$$

Without branes or threshold corrections the dimensionless field $4 \kappa_{4}^{2} C$ is then the (universal) gauge coupling $g_{i}^{2}$.

On the other hand, the chiral version of the theory has gauge kinetic functions (4.17) and then:

$$
\frac{1}{g_{i}^{2}}=\operatorname{Re}\left(S+\beta_{i} T+\tau \hat{\beta}_{i} \hat{S}\right) .
$$

The equality of these two expressions is encoded in the duality transformations exchanging linear multiplets $L$ and $\hat{L}$ with $S$ and $\hat{S}$ :

$$
\begin{aligned}
\frac{1}{2}(\hat{S}+\overline{\hat{S}})= & 2\left(T+\bar{T}-2 \bar{M} e^{2 A} M\right) \hat{V}+2 \sum_{i} \hat{\gamma}_{i} \bar{M}^{i} e^{2 A} M^{i} \\
\frac{1}{2}(S+\bar{S})= & \frac{1}{2}\left(\frac{S_{0} \bar{S}_{0}\left(T+\bar{T}-2 \bar{M} e^{2 A} M\right)}{2 V}\right)^{3 / 2}+\frac{1}{2}\left[\epsilon\left|\alpha M^{3}\right|^{2}-2 \tau \sum_{i} \hat{\delta}_{i} \bar{M}^{i} e^{2 A} M^{i}\right] \\
& +\frac{\tau}{2}\left(T+\bar{T}-2 \bar{M} e^{2 A} M\right) \hat{V}^{2}
\end{aligned}
$$

with $V$ and $\hat{V}$ as in eqs. (4.10). The lowest scalar components of these two superfield equations show the equality of (4.21) and (4.23).

The "natural" definition of the dilaton $\varphi$ with kinetic Lagrangian $\left(\partial_{\mu} \ln \varphi\right)^{2} / 4$ is to identify

$$
-\ln (S+\bar{S}-\Delta) \longleftrightarrow-\ln (2 \varphi)
$$

\footnotetext{
${ }^{22}$ See for instance [36, 13].
} 
in the Kähler potential (4.13), i.e.

$$
\begin{aligned}
\varphi & =\frac{1}{2}\left(\frac{z_{0} \bar{z}_{0}(T+\bar{T}-2 \bar{M} M)}{2 C}\right)^{3 / 2} \\
& =\operatorname{Re} S-\frac{\tau}{32} \frac{\left[\hat{S}+\overline{\hat{S}}-4 \sum_{i} \hat{\gamma}_{i} \bar{M}^{i} M^{i}\right]^{2}}{T+\bar{T}-2 \bar{M} M}+\tau \sum_{i} \hat{\delta}_{i} \bar{M}^{i} M^{i}-\frac{1}{2} \epsilon\left|\alpha M^{3}\right|^{2} .
\end{aligned}
$$

From now on, we omit charged matter terms, as we expect $\langle M\rangle=0$. In terms of the dilaton, the gauge couplings read then

$$
\begin{aligned}
\frac{1}{g_{i}^{2}} & =\varphi+\operatorname{Re} T\left[\tau \hat{C}^{2}+4 \tau \hat{\beta}_{i} \hat{C}+\beta_{i}\right] \\
& =\varphi+\operatorname{Re} T\left[\frac{\tau}{16}\left(\frac{\operatorname{Re} \hat{S}}{\operatorname{Re} T}\right)^{2}+\tau \hat{\beta}_{i} \frac{\operatorname{Re} \hat{S}}{\operatorname{Re} T}+\beta_{i}\right] .
\end{aligned}
$$

They display a universal ${ }^{23}$ correction quadratic in the five-brane location, as well as gauge group-dependent corrections linear in $\hat{C}$ or constant. The chiral version has only terms linear in $S, T$ and $\hat{S}$ : the universal quadratic correction has been absorbed in the non-harmonic redefinition turning the dilaton $\varphi$ into $\operatorname{Re} S$. And of course the quadratic term reappears in the Kähler potential for $S$ [see eqs. (4.13) and (4.16)].

We now restore the summation over several five-branes and split the coefficients $\beta_{i}$ according to $\beta_{i}=\beta_{i}^{(p l .)}+\beta_{i}^{(b r .)} \sum_{I} \tau_{I}$ since they receive in general contributions from both planes and five-branes. ${ }^{24}$ Using the identification (4.19), one obtains

$$
\frac{1}{g_{i}^{2}}=\varphi+\beta_{i}^{(p l .)} \operatorname{Re} T+\operatorname{Re} T \sum_{I} \tau_{I}\left[\beta_{i}^{(b r .)}+\frac{4}{\kappa} \hat{\beta}_{i}\left(\frac{y_{I}}{2 \pi}\right)+\frac{1}{\kappa^{2}}\left(\frac{y_{I}}{2 \pi}\right)^{2}\right] .
$$

Notice that since the exchange $1 \leftrightarrow 2$ of the plane indices is equivalent to moving the five-brane from $y_{I}$ to $\pi-y_{I}$, we expect

$$
\hat{\beta}_{1}+\hat{\beta}_{2}=-\frac{1}{4 \kappa}, \quad \beta_{2}^{(b r .)}=\beta_{1}^{(b r .)}+\frac{2}{\kappa} \hat{\beta}_{1}+\frac{1}{4 \kappa^{2}} .
$$

The next step is to compare these results with the terms obtained from the reduction of the topological term and especially with the brane contributions described by the first term in eq. (3.8):

$$
\begin{gathered}
e_{4}^{-1} \Delta \mathcal{L}_{\text {brane }}=\frac{1}{4} \sum_{I} \frac{\tau_{I}}{\kappa^{2}} \operatorname{Im} T\left\{\left[\left(\frac{y_{I}}{2 \pi}\right)^{2}-\frac{y_{I}}{2 \pi}+\frac{1}{6}\right] \operatorname{Tr} F^{(1)} \widetilde{F}^{(1)}\right. \\
\left.+\left[\left(\frac{y_{I}}{2 \pi}\right)^{2}-\frac{1}{12}\right] \operatorname{Tr} F^{(2)} \widetilde{F}^{(2)}\right\} .
\end{gathered}
$$

\footnotetext{
${ }^{23} i$.e identical for all group factors, all values of index $i$.

${ }^{24}$ The constants $\beta_{i}^{(p l .)}, \beta_{i}^{(b r .)}$ and $\hat{\beta}_{i}$ should not depend on $I$.
} 
The terms of order $\left(y_{I}\right)^{2}$ have identical coefficient for both planes. If our effective supergravity is correct, this contribution should appear in the DBI term appearing in the second line of Lagrangian (4.11). The vector component of the Chern-Simons multiplet $\Omega$ is

$$
[\Omega]_{\text {vector }} \equiv \Omega^{\mu}=\frac{1}{8 e_{4}} \varepsilon^{\mu \nu \rho \sigma} \omega_{\nu \rho \sigma}+\ldots
$$

and the component expansion of (4.11) includes then

$$
-4 e_{4} \tau_{I} \hat{C}_{I}^{2}\left(\partial_{\mu} \operatorname{Im} T\right) \sum_{i} \Omega_{i}^{\mu}+\ldots
$$

Integrating by part for constant values $\hat{C}_{I}=\left\langle\hat{C}_{I}\right\rangle=y_{I} /(2 \pi \kappa)$, we obtain

$$
\frac{1}{4} e_{4} \frac{\tau_{I}}{\kappa^{2}}\left(\frac{y_{I}}{2 \pi}\right)^{2} \operatorname{Im} T \sum_{i} \operatorname{Tr} F_{\mu \nu}^{(i)} \widetilde{F}^{(i) \mu \nu}+\ldots
$$

which fits correctly the quadratic term in (4.28). It is then not needed to perform the Calabi-Yau reduction of the five-brane DBI Lagrangian to find the kinetic terms of the five-brane modulus superfield: knowledge of the superfield structure, eq. (4.11), and of the gauge terms implied by the topological term is sufficient. Similarly, the terms of order $y_{I}$ and $\left(y_{I}\right)^{0}$ in the effective supergravity and in the reduction of the topological term can be used to find the values of the coefficients $\beta_{i}^{(2)}$ and $\hat{\beta}_{i}$.

The second line of Lagrangian (4.11) indicates that the DBI contribution also includes gauge terms of order $\hat{C}$, which after partial integration read

$$
\frac{1}{\kappa} \operatorname{Im} T \sum_{I} \tau_{I} \frac{y_{I}}{2 \pi} \sum_{i} \hat{\beta}_{i} \operatorname{Tr} F_{\mu \nu}^{(i)} \widetilde{F}^{(i) \mu \nu} .
$$

By comparison with (4.28), we find

$$
\hat{\beta}_{1}=-\frac{1}{4 \kappa}, \quad \hat{\beta}_{2}=0 .
$$

Finally, comparison of the $y_{I}$-independent terms in eq. (4.28) with the first term of (4.8), which includes

$$
-2 \sum_{i}\left[\beta_{i} \Omega_{i} V_{T}\right]_{D}=\frac{1}{4} \operatorname{Im} T \sum_{i} \beta_{i} \operatorname{Tr} F_{\mu \nu}^{(i)} \widetilde{F}^{(i) \mu \nu}+\ldots
$$

indicates that

$$
\beta_{1}^{(b r .)}=\frac{1}{6 \kappa^{2}}, \quad \quad \beta_{2}^{(b r .)}=-\frac{1}{12 \kappa^{2}} .
$$

As expected, exchanging planes $1 \leftrightarrow 2$ is equivalent to $y_{I} \leftrightarrow \pi-y_{I}$. 
Finally, as usual, the coefficients $\beta_{i}^{(p l .)}$ can be read directly from the first line of eq. (3.6), which includes contributions to the topological terms arising from the hyperplanes only:

$$
\beta_{i}^{(p l .)}=\frac{2}{(4 \pi)^{4} \kappa^{2}} \sum_{j} d_{i j}\left\langle\mathcal{I}_{j}\right\rangle
$$

or

$$
\beta_{1}^{(p l .)}=\frac{1}{6(4 \pi)^{3} \kappa^{2}}\left(2\left\langle\mathcal{I}_{1}\right\rangle-\left\langle\mathcal{I}_{2}\right\rangle\right), \quad \beta_{2}^{(p l .)}=\frac{1}{6(4 \pi)^{3} \kappa^{2}}\left(2\left\langle\mathcal{I}_{2}\right\rangle-\left\langle\mathcal{I}_{1}\right\rangle\right) .
$$

Notice that

$$
\beta_{1}^{(p l .)}+\beta_{2}^{(p l .)}=\frac{1}{6(4 \pi)^{3} \kappa^{2}}\left(\left\langle\mathcal{I}_{1}\right\rangle+\left\langle\mathcal{I}_{2}\right\rangle\right)
$$

vanishes in the minimal embedding without five-brane [8, 29].

To summarize, in terms of the dilaton, the gauge couplings on both planes, as calculated from the modified topological term, read:

$$
\begin{aligned}
\frac{1}{g_{1}^{2}} & =\varphi+\beta_{1}^{(p l .)} \operatorname{Re} T+\frac{1}{\kappa^{2}} \operatorname{Re} T \sum_{I} \tau_{I}\left[\left(\frac{y_{I}}{2 \pi}\right)^{2}-\left(\frac{y_{I}}{2 \pi}\right)+\frac{1}{6}\right], \\
\frac{1}{g_{2}^{2}} & =\varphi+\beta_{2}^{(p l .)} \operatorname{Re} T+\frac{1}{\kappa^{2}} \operatorname{Re} T \sum_{I} \tau_{I}\left[\left(\frac{y_{I}}{2 \pi}\right)^{2}-\frac{1}{12}\right] .
\end{aligned}
$$

A nicer expression reminiscent of eqs. (2.20) or (2.23) uses the distance from the brane to the planes:

$$
\begin{aligned}
& \frac{1}{g_{1}^{2}}=\varphi+\beta_{1}^{(p l .)} \operatorname{Re} T+\frac{1}{4 \kappa^{2}} \operatorname{Re} T \sum_{I} \tau_{I}\left[\left(\Delta_{I, 2}\right)^{2}-\frac{1}{3}\right] \\
& \frac{1}{g_{2}^{2}}=\varphi+\beta_{2}^{(p l .)} \operatorname{Re} T+\frac{1}{4 \kappa^{2}} \operatorname{Re} T \sum_{I} \tau_{I}\left[\left(\Delta_{I, 1}\right)^{2}-\frac{1}{3}\right] .
\end{aligned}
$$

where $\Delta_{I, 1}=y_{I} / \pi$, and $\Delta_{I, 2}=1-y_{I} / \pi$, as in eq. (2.18). The contribution of a five-brane to the gauge couplings on one hyperplane decreases quadratically from a maximum value

$$
\frac{1}{6 \kappa^{2}} \tau_{I} \operatorname{Re} T
$$

when the brane lies on the plane, to a minimal value

$$
-\frac{1}{12 \kappa^{2}} \tau_{I} \operatorname{Re} T
$$

when the five-brane lies on the opposite plane. For a five-brane in the middle of the interval, both gauge couplings receive the correction

$$
-\frac{1}{48 \kappa^{2}} \tau_{I} \operatorname{Re} T \text {. }
$$


With however $\left(\Delta_{I, i}\right)^{2}=-\Delta_{I, 1} \Delta_{I, 2}+\Delta_{I, i},(i=1,2)$, the term quadratic in $y$ is necessarily universal and the two (inverse squared) gauge couplings differ only by a contribution linear in $y$ :

$$
\frac{1}{g_{1}^{2}}-\frac{1}{g_{2}^{2}}=\frac{\operatorname{Re} T}{2(4 \pi)^{3} \kappa^{2}}\left[\left\langle\mathcal{I}_{1}\right\rangle-\left\langle\mathcal{I}_{2}\right\rangle+\sum_{I} a_{I}\left(1-\frac{2 y_{I}}{\pi}\right)\right],
$$

with $\Delta_{I, 2}-\Delta_{I, 1}=1-2 y_{I} / \pi$ and in terms of the area $a_{I}$ of the two-cycle wrapped by the five-brane in $K_{6}$ [see eq. (4.6)].

The normalization of the four-forms $I_{4,1}$ and $I_{4,2}$ is such that their integrals over a four-cycle in $K_{6}$ are half-integers. Similary $a_{I}$ is an intersection number of the twocycle wrapped by the brane with the four-cycle, in units of the Calabi-Yau volume. ${ }^{25}$ These statements follow from the integrated Bianchi identity verified by $G_{4}$ and from rewriting eqs. (3.7) and (3.9) in the form

$$
a_{I}=-i \int_{K_{6}} \delta^{(4)}\left(\mathcal{W}_{6, I}\right) \wedge d z \wedge d \bar{z}, \quad\left\langle\mathcal{I}_{i}\right\rangle=-i \int_{K_{6}}\left\langle I_{i}\right\rangle \wedge d z d \bar{z}
$$

Eq. (4.38) matches then nicely the idea that a five-brane moved to the hyperplane at $y=0$ decreases the instanton number on this plane, or on the second plane when moved to $y=\pi$.

In the chiral version of the theory, the gauge kinetic functions are

$$
\begin{aligned}
& f_{2}=S+\beta_{2}^{(p l .)} T-\frac{1}{12 \kappa^{2}} \sum_{I} \tau_{I} T \\
& f_{1}=f_{2}+\left[\beta_{1}^{(p l .)}-\beta_{2}^{(p l .)}\right] T+\frac{1}{4 \kappa^{2}} \sum_{I} \tau_{I}[T-\kappa \hat{S}] .
\end{aligned}
$$

Since

$$
\langle\operatorname{Re} T\rangle-\kappa\langle\operatorname{Re} \hat{S}\rangle=\langle\operatorname{Re} T\rangle\left(1-\frac{2 y}{\pi}\right),
$$

the difference is again eq. (4.38).

\subsection{Discussion of some matter terms}

We have seen that the gauge part of the five-brane-induced contributions to the topological terms are due, in the effective supergravity, either to the effect of the modified Bianchi identities on the Dirac-Born-Infeld Lagrangian, or to threshold corrections. Since charged matter multiplets $M$ arise from the fields living on the fixed hyperplanes, as do gauge multiplets, we may expect that some or all matter contributions

\footnotetext{
${ }^{25}$ We have chosen $V_{6}=1$.
} 
obtained from the reduction of the topological term can also be derived from the DBI effective Lagrangian.

As an illustration, we will establish that the charged matter term in expression (3.8) arises for its part quadratic in $y_{I}$ from the DBI Lagrangian, while the terms linear and constant in $y_{I}$ originate from threshold corrections. Since the vector component of $V$ is related to the string antisymmetric tensor $B_{A B}$ by

$$
v^{\mu}=-\frac{2 \pi}{8 e_{4}} \epsilon^{\mu \nu \rho \sigma} \partial_{\nu} B_{\rho \sigma}
$$

the string-matter coupling term in eq. (3.8) takes the form

$$
\begin{gathered}
-\frac{i}{8(4 \pi)^{3}} \sum_{I, i} a_{I}\left(\Delta_{I, i}^{2}-\frac{1}{3}\right) \epsilon^{\mu \nu \rho \sigma} \partial_{\mu} B_{\nu \rho} \sum_{m}\left[\mathcal{A}^{m(i)}\left(\mathcal{D}_{\sigma} \overline{\mathcal{A}}^{m(i)}\right)-\overline{\mathcal{A}}^{m(i)}\left(\mathcal{D}_{\sigma} \mathcal{A}^{m(i)}\right)\right] \\
=-\frac{2 i}{\kappa^{2}} e_{4} \sum_{I} \tau_{I} v^{\mu}\left\{\left(\frac{y_{I}}{2 \pi}\right)^{2}\left[M\left(\mathcal{D}_{\mu} \bar{M}\right)-\bar{M}\left(\mathcal{D}_{\mu} M\right)\right]\right. \\
\left.+\sum_{i} \tilde{g}_{i}\left(y_{I}\right)\left[M^{i}\left(\mathcal{D}_{\mu} \bar{M}^{i}\right)-\bar{M}^{i}\left(\mathcal{D}_{\mu} M^{i}\right)\right]\right\} .
\end{gathered}
$$

The functions $\tilde{g}_{i}\left(y_{I}\right)$ are at most linear in $y_{I}$ :

$$
\tilde{g}_{1}\left(y_{I}\right)=-\frac{y_{I}}{2 \pi}+\frac{1}{6}, \quad \quad \tilde{g}_{2}\left(y_{I}\right)=-\frac{1}{12} .
$$

The first term in the r.h.s. of eq. (4.41) is universal and can clearly be retrieved from the DBI Lagrangian in the second line of theory (4.11) by selecting the matter contribution inside $V_{T}$,

$$
-2 i e_{4} \tau_{I} \hat{C}_{I}^{2} v^{\mu}\left[M\left(\mathcal{D}_{\mu} \bar{M}\right)-\bar{M}\left(\mathcal{D}_{\mu} M\right)\right]+\ldots
$$

and identifying as usual $\hat{C}^{2}$ with $y_{I}^{2}(2 \pi \kappa)^{-2}$.

The second term in the r.h.s. of eq. (4.41) originates from matter threshold corrections in the supergravity Lagrangian (4.11). The relevant term is:

$$
-2 i e_{4} \tau_{I} v^{\mu} \sum_{i} g_{i}(\hat{C})\left[M^{i}\left(\mathcal{D}_{\mu} \bar{M}^{i}\right)-\bar{M}^{i}\left(\mathcal{D}_{\mu} M^{i}\right)\right]+\ldots
$$

Comparison with eq. (4.41) indicates that

$$
\hat{\gamma}_{1}=-\frac{1}{\kappa} \quad \hat{\gamma}_{2}=0, \quad \hat{\delta}_{1}=\frac{1}{6 \kappa^{2}}, \quad \hat{\delta}_{2}=-\frac{1}{12 \kappa^{2}} .
$$

Interestingly enough, $\hat{\delta}_{i}=\beta_{i}^{(b r .)}$ and $\hat{\gamma}_{i}=4 \hat{\beta}_{i}$. 
Finally, we briefly return to the issue of tracing back the supersymmetric origin of interactions such as expressions (3.11) and (3.12). As already mentioned in Section 3, eq. (3.11) seems in general hard to cast in a supersymmetric form because of the complicated mixing of matter contributions from both hyperplanes. This feature is also present in eq. (3.12), forbidding by the same token its supersymmetrization for a generic background. We however have little information on the nature of fourdimensional matter counter-terms which could be added to anomaly-cancelling corrections and could radically change the picture. At this level of understanding, this discussion cannot be conclusive.

Nevertheless, eq. (3.11) allows a supersymmetric formulation for the standard embedding in the gauge group in which chiral matter multiplets only appear on one plane, say $\mathcal{A} \equiv \mathcal{A}_{2}$. Then, eq. (3.11) reduces to

$$
\begin{aligned}
\Delta \mathcal{L}_{\text {plane }}= & i \epsilon^{\mu \nu \rho \sigma} \omega_{1, \mu \nu \rho}\left\{\frac{1}{3(4 \pi)^{5}}\left[\lambda \mathcal{A}^{3} \partial_{\sigma}\left(\overline{\lambda \mathcal{A}}^{3}\right)-\overline{\lambda \mathcal{A}}^{3} \partial_{\sigma}\left(\lambda \mathcal{A}^{3}\right)\right]\right. \\
& \left.-\frac{1}{12(4 \pi)^{3}} \sum_{m}\left(\mathcal{A}^{m} \mathcal{D}_{\sigma} \overline{\mathcal{A}}^{m}-\overline{\mathcal{A}}^{m} \mathcal{D}_{\sigma} \mathcal{A}^{m}\right)\left(\left\langle\mathcal{I}_{1}\right\rangle-\left\langle\mathcal{I}_{2}\right\rangle\right)\right\}
\end{aligned}
$$

which extends to the supersymmetric density

$$
\frac{1}{3(4 \pi)^{2} \kappa^{6}}\left[\Omega_{1}\left|\alpha M^{3}\right|^{2}\right]_{D}-\frac{1}{3(4 \pi)^{2} \kappa^{2}}\left(\left\langle\mathcal{I}_{1}\right\rangle-\left\langle\mathcal{I}_{2}\right\rangle\right)\left[\Omega_{1} \bar{M} e^{2 A} M\right]_{D} .
$$

The second term could in principle correspond to the first contribution appearing in the threshold correction (4.8).

\section{Condensation, the non-perturbative superpoten- tial and membrane instantons}

The non-perturbative superpotential arises from the $F$-density in the supergravity Lagrangian (4.3) when some or all gauge fields condense. It can be evaluated using a standard two-step procedure: first obtain the effective action for condensates and then eliminate the condensate by solving (approximately in general) its field equation. Computing the effective action amounts in principle to couple the superfield $\mathcal{W} \mathcal{W}$ to an external source, integrate the gauge fields and perform the Legendre transformation exchanging the source field with the (classical) condensate field. It is well known that the symmetry content of super-Yang-Mills theory and anomaly-matching are sufficiently restrictive to accurately describe condensation [37].

As usual, we assume that the gauge multiplet which first condenses does not couple to matter multiplets $M$. We then split the gauge group into $\mathcal{G}_{0} \times \prod_{a} \mathcal{G}_{a}$, where the 
simple group $\mathcal{G}_{0}$ condenses and matter multiplets only tranform under $\prod_{a} \mathcal{G}_{a}$. The terms involving $\mathcal{G}_{0}$ gauge fields in the Lagrangian reduce then to

$$
\frac{1}{4}\left[\left(S+\beta_{0} T+\tau \hat{\beta}_{0} \hat{S}\right) \mathcal{W}_{0} \mathcal{W}_{0}\right]_{F} .
$$

Following ref. [37, these contributions are simply replaced in the effective action for condensates by

$$
\frac{1}{4}\left[\left(S+\beta_{0} T+\tau \hat{\beta}_{0} \hat{S}\right) U+\frac{b_{0}}{24 \pi^{2}}\left\{U \ln \left(\frac{U}{\mu^{3}}\right)-U\right\}\right]_{F}+\left[S_{0} \bar{S}_{0} \widetilde{K}\left(U S_{0}^{-3}, \overline{U S}_{0}^{-3}\right)\right]_{D}
$$

where $U$ is the (classical) chiral superfield $(w=n=3)$ describing the gaugino condensate, $\langle U\rangle=\langle\lambda \lambda\rangle$. The coefficient of the Veneziano-Yankielowicz superpotential is dictated by the anomaly of the superconformal chiral $U(1)$. It is proportional to the one-loop beta function, $b_{0}=3 C\left(\mathcal{G}_{0}\right)$, and the scale parameter $\mu$ is the energy at which gauge couplings in expressions (4.37) are defined. Finally, the leading contribution to the Kähler potential $\widetilde{K}$ is controlled by the scaling dimension (Weyl weight) of $U$.

The effective Lagrangian with condensate $U$ can be obtained by collecting all terms in the "microscopic" Lagrangian (4.3), with contributions (5.1) replaced by the effective terms (15.2):

$$
\begin{aligned}
\mathcal{L}_{e f f .}= & {\left[-\left\{S_{0} \bar{S}_{0}\left(T+\bar{T}-2 \bar{M} e^{2 A} M\right)\right\}^{3 / 2}(2 V)^{-1 / 2}-(S+\bar{S}) V\right.} \\
& -\tau V \hat{V}^{2}\left(T+\bar{T}-2 \bar{M} e^{2 A} M\right)+\frac{\tau}{2}(\hat{S}+\overline{\hat{S}}) V \hat{V} \\
& \left.+V\left\{\epsilon\left|\alpha M^{3}\right|^{2}-2 \tau \sum_{i} g_{i}(\hat{V}) \bar{M}^{i} e^{2 A} M^{i}\right\}+S_{0} \bar{S}_{0} \widetilde{K}\left(U S_{0}^{-3}, \overline{U S_{0}^{-3}}\right)\right]_{D} \\
& +\frac{1}{4}\left[\sum_{a}\left(S+\beta_{a} T+\tau \hat{\beta}_{a} \hat{S}\right) \mathcal{W}_{a} \mathcal{W}_{a}\right]_{F} \\
& +\left[S_{0}^{3}\left(c+\alpha M^{3}\right)+\frac{1}{4}\left(S+\beta_{0} T+\tau \hat{\beta}_{0} \hat{S}\right) U+\frac{C\left(\mathcal{G}_{0}\right)}{32 \pi^{2}}\left\{U \ln \left(U / \mu^{3}\right)-U\right\}\right]_{F} .
\end{aligned}
$$

As before, vector multiplets $V$ and $\hat{V}$ are constrained by the field equations of Lagrange multipliers $S$ and $\hat{S}$, which impose modified Bianchi identities. Rewriting their contributions in the form

$$
\left[2 S\left\{\Sigma(V)+\frac{1}{8} U+\frac{1}{8} \sum_{a} \mathcal{W}_{a} \mathcal{W}_{a}\right\}-\tau \hat{S}\left\{\Sigma(V \hat{V})-\frac{1}{4} \hat{\beta}_{0} U-\frac{1}{4} \sum_{a} \hat{\beta}_{a} \mathcal{W}_{a} \mathcal{W}_{a}\right\}\right]_{F}
$$

multiplier $S$ implies

$$
U=-8 \Sigma\left(V_{0}\right), \quad V=V_{0}-2 \sum_{a} \Omega_{a}
$$


with a real vector multiplet $V_{0}$ ( 8 bosons +8 fermions $)$. Then, multiplier $\hat{S}$ requires

$$
V \hat{V}=\hat{L}_{0}-2 \hat{\beta}_{0} V_{0}+4 \sum_{a} \hat{\beta}_{a} \Omega_{a}
$$

with a linear multiplet $\hat{L}_{0}$ and $V$ as given in eqs. (5.5). These solutions can be compared with eqs. (4.10), which apply before condensation of $\mathcal{W}_{0} \mathcal{W}_{0}$. Clearly, the real vector multiplet $V_{0}$ describes $L-\left.2 \Omega_{0}\right|_{\text {cond. }}$, i.e. it includes the string coupling linear multiplet $L$ and the condensate field 31. Similarly, the linear multiplet $\hat{L}_{0}$ replaces $\hat{L}+2 \hat{\beta}_{0} L$.

To obtain the non-perturbative superpotential however, one first chooses the formulation of the theory with chiral multiplets only. The elimination of vector multiplets $V$ and $\hat{V}$ is as in the previous section. Omitting from here on gauge fields $\mathcal{W}_{a}$ and charged matter fields $M$, the chiral formulation of the effective Lagrangian is

$$
\begin{aligned}
\mathcal{L}_{\text {eff. }}= & {\left[-\frac{3}{2} S_{0} \bar{S}_{0} e^{-\mathcal{K} / 3}+S_{0} \bar{S}_{0} \widetilde{K}\right]_{D} } \\
& +\left[S_{0}^{3}\left(c+\alpha M^{3}\right)+\frac{1}{4}\left(S+\beta_{0} T+\tau \hat{\beta}_{0} \hat{S}\right) U+\frac{C\left(\mathcal{G}_{0}\right)}{32 \pi^{2}}\left\{U \ln \left(U / \mu^{3}\right)-U\right\}\right]_{F} .
\end{aligned}
$$

The Kähler potential $\mathcal{K}$ is as in eqs. (4.13) and (4.16), with full mixing of multiplets $S, T$ and $\hat{S}$. To derive the non-perturbative effective potential, neglect $\widetilde{K} \cdot{ }^{26}$ The field equation of the condensate field $U$ implies then

$$
U=\mu^{3} \exp \left(-\frac{8 \pi^{2}}{C\left(\mathcal{G}_{0}\right)}\left[S+\beta_{0} T+\tau \hat{\beta}_{0} \hat{S}\right]\right) \equiv \mathcal{U},
$$

and the effective superpotential becomes

$$
W_{n p}=c+\alpha M^{3}-\frac{C\left(\mathcal{G}_{0}\right)}{32 \pi^{2}} \mathcal{U} S_{0}^{-3} .
$$

This superpotential is the sum of the 'microscopic' superpotential and the non-perturbative contribution of the gauge condensate. The non-perturbative contribution is the exponential of the sum of the string coupling, Calabi-Yau volume and five-brane moduli contributions. With several condensates, the non-perturbative piece would be replaced by a sum of similar terms over all gauge simple factors which condense.

To get a qualitative picture of the effect of the five-brane, use eqs. (4.39) and (4.38) to rewrite the condensate as a function of the five-brane position along $S^{1}$, assuming first that the condensate arises on the hyperplane at $y=0$ :

$$
\begin{aligned}
|U|_{1 \text { st plane }} & =|\mu|^{3} \exp \left(-\frac{8 \pi^{2}}{C\left(\mathcal{G}_{0}\right)}\left[\operatorname{Re} S+\frac{1}{(4 \pi)^{3} \kappa^{2}} \operatorname{Re} T\left\{\frac{1}{3}\left\langle\mathcal{I}_{1}\right\rangle-\frac{1}{6}\left\langle\mathcal{I}_{2}\right\rangle+\frac{1}{3} a-\frac{y}{\pi} a\right\}\right]\right) \\
& =|\mu|^{3} \exp \left(-\frac{8 \pi^{2}}{C\left(\mathcal{G}_{0}\right) g_{1}^{2}}\right) .
\end{aligned}
$$

${ }^{26}$ Disregarding the Kähler potential $\widetilde{K}$ is the same as considering $U$ as a constant background field with value chosen to extremize the action. 
If the condensate arises on the hyperplane at $y=\pi$ :

$$
\begin{aligned}
|U|_{2 \text { nd plane }} & =|\mu|^{3} \exp \left(-\frac{8 \pi^{2}}{C\left(\mathcal{G}_{0}\right) g_{2}^{2}}\right) \\
& =|U|_{1 \text { st plane }} \times \exp \left(\frac{4 \pi^{2}}{C\left(\mathcal{G}_{0}\right)} \frac{\operatorname{Re} T}{(4 \pi)^{3} \kappa^{2}}\left[\left\langle\mathcal{I}_{1}\right\rangle-\left\langle\mathcal{I}_{2}\right\rangle+a\left(1-\frac{2 y}{\pi}\right)\right]\right)
\end{aligned}
$$

The non-perturbative superpotential (5.9) and the condensates (5.10) and (5.11) display the dependence on the five-brane location on $S^{1}$ expected from explicit calculations of membrane instanton corrections in the four-dimensional effective theory 14. 18. We have obtained this dependence from the analysis of the fundamental Bianchi identity of M-theory and from the (modified) topological term, showing in this way that open membrane instanton contributions find their higher-dimensional origin in anomaly-cancellation in the presence of five-branes.

This observation has a second consequence. The non-perturbative superpotential is not a sum of exponential terms generated by gaugino condensates and membrane instantons, a structure which is not in any case expected to appear in the effective supergravity. Instead, we find a non-perturbative term which is the exponential of a sum of terms linear in the chiral fields, a structure characteristic of threshold corrections induced by anomaly-cancellation in higher dimensions.

In our reduction scheme, the "microscopic" superpotential $c+\alpha M^{3}$ is moduliindependent. It is however known that T-duality induces a holomorphic dependence on $T$ compatible with our supermultiplet structure as described in Lagrangian (5.3). The existence of dual descriptions of moduli $S$ and $\hat{S}$ in terms of constrained vector multiplets $V$ and $\hat{V}$ or in terms of linear multiplets $L$ and $\hat{L}$ implies that the "microscopic" superpotential cannot depend on $S$ or $\hat{S}$.

\subsection{The scalar potential}

Because of the mixing of the three moduli multiplets $S, T$ and $\hat{S}$ in the Kähler metric, the scalar potential present in the component expansion of the effective Lagrangian (5.3) is not positive and analysing its vacuum structure is a severe problem. This mixing is due to the kinetic terms of the five-brane massless modes, it is unavoidable whenever five-branes, and then whenever $\hat{S}$, are present.

We may however gain insight by deriving the scalar potential directly in terms of the components of the constrained vector multiplets. This version of the theory is indeed more tractable than the chiral one since the mixing of moduli fields is simpler. The relevant multiplets are then $T, V, \hat{V}$ for the moduli and $U$ for the condensate. 
Charged matter terms are as before omitted since we are interested in vacua where they vanish. In the Einstein frame (4.22), the relation between the dilaton (4.25) and the lowest scalar component $C$ of $V$ is

$$
\varphi=\frac{1}{4 \kappa_{4}^{2} C}
$$

and the scalar potential is eventually expressed in terms of the physical dilaton. As explained in eqs. (5.5), eliminating $S$ with $\Omega_{a}$ omitted generates the modified Bianchi identity $U=-8 \Sigma(V)$, where $V$ is the vector field describing $L-\left.2 \Omega_{0}\right|_{\text {cond. }}$. To derive the potential, we only need the scalar components of $V$ and $U$,

$$
\begin{array}{ll}
V=(C, 0, H, K, 0,0, d), & U=\left(u, 0, f_{U}\right), \\
u=-4(H-i K), & f_{U}=4 d .
\end{array}
$$

Since a non-zero condensate $u$ also switches on the field $H-i K$ of the dilaton multiplet $V$, the gaugino condensate clearly breaks supersymmetry in this sector, as expected in a theory where the dilaton couples to gauge fields. Eliminating $\hat{S}$ defines the five-brane (effective) linear multiplet $V \hat{V}=\hat{L}_{0}-2 \hat{\beta}_{0} V$ and, since linear multiplets do not have auxiliary fields, we may simply write

$$
\hat{L}_{0}=\left(C \hat{C}+2 \hat{\beta}_{0} C, 0,0,0,0,0,0\right),
$$

in terms of the lowest scalar component $\hat{C}$ of $\hat{V}$, when deriving the scalar potential. The resulting scalar potential is then a function of the physical scalars $C$ (the dilaton, see. eq. 5.12), the $S^{1} / Z_{2}$ modulus $\hat{C}=y /(2 \pi \kappa)$, the Calabi-Yau volume modulus $T$ and the gaugino condensate $u$. It is also a function of the auxiliary fields $d, f_{T}$ and $f_{0}$ (in the compensating multiplet $S_{0}$ ) which can be easily eliminated.

The Kähler potential $\widetilde{K}$ generates a term quadratic in $d$. We will write the scalar potential by restricting $\widetilde{K}$ to its leading term 37]

$$
\widetilde{K}\left(U S_{0}^{-3}, \overline{U S}_{0}^{-3}\right) S_{0} \bar{S}_{0}=A(U \bar{U})^{1 / 3}
$$

with an arbitrary normalisation constant $A \geq 0$. The scalar potential as a function of 
$d, C, \hat{C}, T$ and $u$ reads then:

$$
\begin{aligned}
V_{\text {eff. }}= & -\frac{32}{9} A(u \bar{u})^{-2 / 3} d^{2}-B d \\
& +\frac{u \bar{u}}{16 C}\left[\frac{3}{2} \frac{1}{4 \kappa_{4}^{2} C}+\tau\left(\hat{C}+2 \hat{\beta}_{0}\right)^{2}(T+\bar{T})\right] \\
& +\frac{2 \kappa_{4}^{2} C}{\kappa_{4}^{4}(T+\bar{T})^{3}}\left\{-2\left|W+\frac{1}{8} \kappa_{4}^{3} u\left(\frac{T+\bar{T}}{2 \kappa_{4}^{2} C}\right)^{3 / 2}\right|^{2}\right. \\
& \left.+\frac{(T+\bar{T})^{2}}{3}\left|W_{T}-\frac{3}{T+\bar{T}} W+\frac{1}{4} \kappa_{4}^{3} u \frac{(T+\bar{T})^{3 / 2}}{\left(2 \kappa_{4}^{2} C\right)^{1 / 2}}\left(\beta_{0}+\tau\left[\hat{C}^{2}+4 \hat{\beta}_{0} \hat{C}\right]\right)\right|^{2}\right\}
\end{aligned}
$$

The first two terms arise respectively from the condensate Kähler potential term $\left[S_{0} \bar{S}_{0} \widetilde{K}\right]_{D}=A\left[(U \bar{U})^{1 / 3}\right]_{D}$ and from the condensate $F$-density

$$
\left[W_{\text {cond. }}\right]_{F} \equiv\left[\frac{1}{4}\left(S+\beta_{0} T+\tau \hat{\beta}_{0} \hat{S}\right) U+\frac{b_{0}}{96 \pi^{2}}\left\{U \ln \left(U / \mu^{3}\right)-U\right\}\right]_{F} .
$$

The coefficient $B$ relates the gaugino condensate field $u$ and the gauge coupling $g_{0}^{2}$, as defined in eq. (4.26):

$$
B=\frac{2}{g_{0}^{2}}+\frac{b_{0}}{24 \pi^{2}} \ln \left(\frac{u \bar{u}}{\mu^{6}}\right)=8 \operatorname{Re} \frac{\partial}{\partial u} W_{\text {cond. }} .
$$

The standard field-theory value of the condensate,

$$
|u|=\mu^{3} \exp \left(-\frac{24 \pi^{2}}{b_{0} g_{0}^{2}}\right),
$$

is obtained if $B=0$ is part of the scalar potential vacuum equations.

In eq. (15.15), the second line is proportional to $|H-i K|^{2}$ and the fourth line to $\left|f_{T}\right|^{2}$, and we have included the possibility of a $T$-dependent perturbative superpotential, as often implied by $T$-duality, even if our reduction scheme predicts $W_{T}=\frac{\partial W}{\partial T}=0$. The dependence of the potential on the five-brane position $\hat{C}=y /(2 \pi \kappa)$ is best understood by defining the distance $\Delta_{c}$ from the brane to the condensate:

$$
\begin{array}{ll}
\text { For a condensate on plane 1: } & \Delta_{c}=y / \pi=2 \kappa \hat{C} . \\
\text { For a condensate on plane 2: } & \Delta_{c}=1-y / \pi=1-2 \kappa \hat{C} .
\end{array}
$$

Using then the values of the threshold coefficients found in eqs. (4.31) and (4.33), we find in both cases:

$$
\begin{aligned}
\beta_{0}+\tau\left[\hat{C}^{2}+4 \hat{\beta}_{0} \hat{C}\right] & =\beta_{0}^{(p l .)}+\frac{\tau}{4 \kappa^{2}}\left[\left(1-\Delta_{c}\right)^{2}-\frac{1}{3}\right] \\
\left(\hat{C}+2 \hat{\beta}_{0}\right)^{2} & =\frac{1}{4 \kappa^{2}}\left(1-\Delta_{c}\right)^{2}
\end{aligned}
$$


These results agree with the dependence on the five-brane location found in gauge couplings (4.37).

At this stage, we have two options. We may neglect the Kähler potential $\widetilde{K}$ and assume $A=0$. Then, the auxiliary field $d$ imposes the field equation $B=0$ and the correct value of the gaugino condensate. This procedure is equivalent to the derivation of the effective non-perturbative superpotential (5.9). The field equation $B=0$ allows to eliminate $u$ and to express the effective potential, which does not include the first line in expression (5.15), as a function of the moduli scalars $C, \hat{C}$ and $T$ only.

Instead, with a non-zero Kähler potential $\widetilde{K}$ (i.e. with $A>0$ ), solving for the auxiliary $d$ turns the first two terms of the scalar potential (5.15) into

$$
\frac{9}{128} \frac{B^{2}}{A}(u \bar{u})^{2 / 3},
$$

and a generic (non supersymmetric) stationary point of the potential does not necessarily lead to $B=0$ and to the standard gaugino condensate (5.17). But since $B$ appears quadratically in the potential, the same stationary points with $B=0$ would exist in both cases $A=0$ and $A>0$. Notice that the condensate term (5.20) can also be written

$$
\frac{1}{2}\left(\widetilde{K}_{u \bar{u}}\right)^{-1}\left(\operatorname{Re} \frac{\partial}{\partial u} W_{\text {cond. }}\right)^{2}, \quad \quad \widetilde{K}_{u \bar{u}}=\frac{\partial^{2}}{\partial u \partial \bar{u}} A(u \bar{u})^{1 / 3} .
$$

This is the potential term due to the auxiliary field $f_{u}$ of the condensate chiral superfield $U$, with non-standard Weyl weight $w=3$. The imaginary part of $\frac{\partial}{\partial u} W_{\text {cond. }}$ does not contribute to the potential because of the constraint $U=-8 \Sigma(V)$ [31].

A complete analysis of the stationary values of the scalar potential (5.15) cannot be performed analytically. In the absence of five-branes, the potential can be written in the form

$$
V=\frac{1}{2}\left(\widetilde{K}_{u \bar{u}}\right)^{-1}\left(\operatorname{Re} \frac{\partial}{\partial u} W_{\text {cond. }}\right)^{2}+\frac{1}{\kappa_{4}^{2}}\left[\left(2 \kappa_{4}^{2} C\right)^{2}\left|f_{S}\right|^{2}+3(T+\bar{T})^{-2}\left|f_{T}\right|^{2}\right]-\frac{3}{\kappa_{4}^{4}} e^{\mathcal{K}}|W|^{2},
$$

in terms of the Kähler potential $\mathcal{K}=-\ln (S+\bar{S})-3 \ln (T+\bar{T})$ with diagonal metric. A relatively simple study of the stationary points of the potential with for instance $B=0$ can be performed as a function of the auxiliary fields $f_{S}$ and $f_{T}$ of the chiral dilaton $S$ and the volume modulus $T$ respectively.

But the introduction of the five-brane mode leads to mixings of the chiral superfields. For instance, according to the second superfield eq. (4.24), the auxiliary field in the chiral dilaton multiplet $S$ reads

$$
\begin{aligned}
f_{S}= & \kappa_{4}^{-1}\left(2 \kappa_{4}^{2} C\right)^{-1 / 2}(T+\bar{T})^{-3 / 2}\left[\bar{W}-\frac{1}{4}\left(\frac{T+\bar{T}}{2 \kappa_{4}^{2} C}\right)^{3 / 2} \kappa_{4}^{3} \bar{u}\right] \\
& +\tau\left[\hat{C}^{2} f_{T}-\frac{1}{2}(T+\bar{T})\left(\hat{C}^{2}+2 \hat{\beta}_{0} \hat{C}\right)\left(2 \kappa_{4}^{2} C\right)^{-1} \kappa_{4}^{2} \bar{u}\right] .
\end{aligned}
$$


The second term is due to the five-brane and it involves the auxiliary field $f_{T}$, which is proportional to the last line in the potential (5.15). Similarly, the auxiliary component of the five-brane multiplet $\hat{S}$ is

$$
f_{\hat{S}}=4 \hat{C} f_{T}-(T+\bar{T})\left(\hat{C}+2 \hat{\beta}_{0}\right)\left(2 \kappa_{4}^{2} C\right)^{-1} \kappa_{4}^{2} \bar{u}
$$

Mixings of the auxiliary fields then arise whenever $\hat{C} \neq 0$, i.e. whenever the five-brane does not lie on the fixed hyperplane at $y=0$.

\section{Conclusion}

In this paper, we have studied the Calabi-Yau reduction of the low-energy limit of M-theory on the interval $S^{1} / Z_{2}$, with five-branes aligned to preserve four supercharges.

We have used a fully consistent, four-dimensional supergravity and superfield setup and included the modulus field describing five-brane fluctuations in the interval direction. The gauge anomaly-cancelling topological term is modified as a consequence of the five-brane contributions to M-theory Bianchi identities. We have derived the new fourdimensional interactions induced by these five-brane modifications and shown that they lead to new gauge threshold corrections with a calculable dependence on the fivebrane position along $S^{1}$. In particular, these threshold corrections fit nicely the change in the instanton number expected when a five-brane is moved onto one of the fixed hyperplanes. Of course, when gauge condensation occurs, these threshold corrections explicitly appear in the effective non-perturbative superpotential, with an exponential dependence of the five-brane location.

The same five-brane-dependent contributions to the non-perturbative superpotential can be obtained from a different perspective. It is expected that open membranes wrapping a two-cycle in the Calabi-Yau threefold and extending from a five-brane to a ten-dimensional hyperplane generate, in the four-dimensional effective field theory, instanton-like contributions. Instanton calculus allows to explicitly compute these instanton corrections and the resulting contributions to the non-perturbative effective superpotential. Strictly speaking however, instanton calculus only applies in specific limits, which in the case under scrutiny restricts the understanding of the global structure of the superpotential and of the interplay of the various moduli fields. It is precisely here that our effective supergravity Lagrangian, as derived from M-theory, adds important new information. In particular, since membrane instanton corrections actually originate from threshold corrections related to ten-dimensional anomaly cancellation, the non-perturbative superpotential is the exponential of a sum of terms linear in moduli chiral fields. 


\section{Acknowledgements}

We thank Adel Bilal and Claudio Scrucca for helpful discussions. This work has been supported by the Swiss National Science Foundation and by the Commission of the European Communities under contract MRTN-CT-2004-005104.

\section{Appendix A: conventions and notations}

Our conventions are as in refs. [26] and [25]. We use the upstairs picture $M_{4} \times K_{6} \times$ $S^{1} / Z_{2}$, where the $S^{1}$ coordinate is $x^{10}=y R$, with a $(2 \pi)$-periodic angular variable $y$. We use $-\pi<y \leq \pi$ when explicit values are needed because of the natural action of $Z_{2}$ in this interval. Our indices convention for the $M_{4} \times K_{6} \times S^{1} / Z_{2}$ reduction is

$$
x^{M}=\left(x^{A}, y R\right)=\left(x^{\mu}, z^{i}, \bar{z}^{\bar{k}}, y R\right), \quad M=0, \ldots, 10, \quad A=0, \ldots, 9, \quad i, \bar{k}=1,2,3 .
$$

For bulk moduli, we use the terminology familiar from string compactifications: the moduli $s=\operatorname{Re} S$ and $t=\operatorname{Re} T$ with Kähler potential (3.1) are respectively related to the dilaton (or string coupling) and to the Calabi-Yau volume. This convention follows from the metric

$$
d s_{11}^{2}=e^{-2 \phi / 3} g_{A B}^{(10)} d x^{A} d x^{B}+e^{4 \phi / 3} d y^{2},
$$

which defines the string frame and the string coupling $e^{-2 \phi}$ with $R=e^{2 \phi / 3}$, together with

$$
g_{A B}^{(10)} d x^{A} d x^{B}=g_{\mu \nu} d x^{\mu} d x^{\nu}+V^{1 / 3} \delta_{i \bar{j}} d z^{i} d \bar{z}^{\bar{j}}
$$

which defines the Calabi-Yau volume. Rescaling $g_{\mu \nu}$ to the four-dimensional Einstein frame leads to

$$
d s_{11}^{2}=e^{4 \phi / 3}\left[V^{-1} g_{\mu \nu} d x^{\mu} d x^{\nu}+d y^{2}\right]+e^{-2 \phi / 3} V^{1 / 3} \delta_{i \bar{j}} d z^{i} d z^{\bar{j}}
$$

Comparison with the standard eleven-dimensional metric used to diagonalize kinetic terms in the four-dimensional $\mathcal{N}=1$ supergravity Lagrangian,

$$
d s_{11}^{2}=s^{-2 / 3}\left[t^{-1} g_{\mu \nu} d x^{\mu} d x^{\nu}+t^{2} d y^{2}\right]+s^{1 / 3} \delta_{i \bar{j}} d z^{i} d z^{\bar{j}}
$$

leads to the identifications

$$
(\operatorname{Re} T)^{3}=V, \quad \operatorname{Re} S=V e^{-2 \phi}
$$


Hence, $t$ is the volume modulus while $s$ is the dilaton or string coupling modulus.

The terminology often used in the context of M-theory defines instead another Calabi-Yau volume $\hat{V}$ in units specified by the metric (A.3), with then

$$
\hat{V} \equiv V e^{-2 \phi}=\operatorname{Re} S, \quad(\operatorname{Re} T)^{3}=\hat{V} e^{2 \phi}=\hat{V} R^{3}
$$

It seemingly exchanges the respective roles of the bulk moduli.

In order to avoid duplication of contributions due to $Z_{2}$ periodicity, our elevendimensional supergravity action and Green-Schwarz terms are multiplied by $1 / 2$ with respect to standard conventions in use for $M_{11}$ :

$$
\mathcal{L}_{\text {C.J.S. }}+\mathcal{L}_{G . S}=\frac{1}{4 \kappa^{2}}\left[e R-\frac{1}{2} G_{4} \wedge * G_{4}-\frac{1}{6} C_{3} \wedge G_{4} \wedge G_{4}\right]-\frac{T_{2}}{4 \pi} G_{4} \wedge X_{7},
$$

with membrane tension $T_{2}=2 \pi\left(4 \pi \kappa^{2}\right)^{-1 / 3}$, and

$$
d X_{7}=X_{8}=\frac{1}{(2 \pi)^{3} 4 !}\left[\frac{1}{8} \operatorname{tr} R^{4}-\frac{1}{32}\left(\operatorname{tr} R^{2}\right)^{2}\right]
$$

To respect $Z_{2}$ symmetry, we assume that a five-brane with world-volume located at some $y_{0}$ has a $Z_{2}-$ mirror at $-y_{0}$. The Dirac distribution transverse to its world-volume $M_{4} \subset \mathcal{W}_{6} \subset M_{4} \times K_{6}$ is then defined by the condition

$$
\int_{M_{4} \times K_{6} \times S^{1} / Z_{2}} I_{6} \wedge \delta^{(5)}\left(\mathcal{W}_{6}\right)=2 \int_{\mathcal{W}_{6}} I_{6}
$$

for any 6 -form $I_{6}$, since it takes both copies into account.

The membrane and five-brane tensions $T_{2}$ and $T_{5}$ are related by the Dirac-Zwanziger quantization condition

$$
2 \kappa^{2} T_{2} T_{5}=2 \pi
$$

and also by 38

$$
\left(T_{2}\right)^{2}=2 \pi T_{5}
$$

We then express all constants in terms of $\kappa^{2}$, with

$$
\left(4 \pi \kappa^{2}\right)^{1 / 3}=\frac{2 \pi}{T_{2}}=2 \kappa^{2} T_{5} .
$$

With these conventions, the Bianchi identity is

$$
d G_{4}=\frac{4 \pi}{T_{2}}\left(\frac{1}{2} \sum_{I} q_{I} \delta^{(5)}\left(\mathcal{W}_{6, I}\right)-\sum_{i} \delta_{i} I_{4, i}\right)
$$

where the index $I$ labels the $Z_{2}$-symmetric pairs of five-branes and the charge $q_{I}$ is +1 for a five-brane, -1 for an anti-five-brane. 
One subtlety when integrating the Bianchi identity is that one cannot find a $Z_{2}$-odd function $\epsilon(y)$ such that $d \epsilon=\delta\left(y-y_{0}\right) d y$. As in ref. [25], we then use $\left.y, y_{0} \in\right]-\pi, \pi$ ] and

$$
\begin{array}{ll}
\epsilon_{y_{0}}(y)=\operatorname{sgn}\left(y-y_{0}\right)-\frac{y-y_{0}}{\pi}, & d \epsilon_{y_{0}}(y)=\left(2 \delta\left(y-y_{0}\right)-\frac{1}{\pi}\right) d y, \\
\epsilon_{1}(y)=\epsilon_{0}(y)=\operatorname{sgn}(y)-\frac{y}{\pi}, & d \epsilon_{1}(y)=2 \delta_{1}-\frac{1}{\pi} d y, \\
\epsilon_{1}(y)=\epsilon_{\pi}(y)=\operatorname{sgn}(y-\pi)-\frac{y-\pi}{\pi}, & d \epsilon_{2}(y)=2 \delta_{2}-\frac{1}{\pi} d y .
\end{array}
$$

The sign function is

$$
\operatorname{sgn}(r)=\frac{r}{|r|} \quad \text { if } \quad r \neq 0, \quad \operatorname{sgn}(0)=0, \quad \operatorname{sgn}(r)=-\operatorname{sgn}(-r) .
$$

With this definition, $\epsilon_{1}$ and $\epsilon_{2}$ are odd functions while $\epsilon_{y_{0}}(-y)=-\epsilon_{-y_{0}}(y)$. The function

$$
\epsilon_{y_{0}}(y)+\epsilon_{-y_{0}}(y)=\operatorname{sgn}\left(y-y_{0}\right)+\operatorname{sgn}\left(y+y_{0}\right)-\frac{2 y}{\pi}
$$

$\left(0<y_{0}<\pi\right)$ is then odd with

$$
d\left[\epsilon_{y_{0}}(y)+\epsilon_{-y_{0}}(y)\right]=2\left(\delta\left(y-y_{0}\right)+\delta\left(y+y_{0}\right)\right) d y-\frac{2 d y}{\pi} .
$$

This function is useful to insert five-brane sources in the Bianchi identity. Since the five-brane world-volumes $\mathcal{W}_{6, I}$ are of the form $M_{4} \times \mathcal{C}_{2}\left(\mathcal{C}_{2}\right.$ a holomorphic cycle in $\left.K_{6}\right)$, $\mathcal{W}_{6, I}$ is located at $y=y_{I}$ with a " $Z_{2}-$ mirror five-brane" at $-y_{I}$. We then use

$$
\begin{aligned}
& \delta^{(5)}\left(\mathcal{W}_{6, I}\right)=\left(\delta\left(y-y_{I}\right)+\delta\left(y+y_{I}\right)\right) d y \wedge \delta^{(4)}\left(\mathcal{W}_{6, I}\right) \\
& d\left(\left[\epsilon_{y_{I}}(y)+\epsilon_{-y_{I}}(y)\right] \delta^{(4)}\left(\mathcal{W}_{6, I}\right)\right)=2 \delta^{(5)}\left(\mathcal{W}_{6, I}\right)-\frac{2}{\pi} d y \wedge \delta^{(4)}\left(\mathcal{W}_{6, I}\right),
\end{aligned}
$$

to integrate five-brane contributions to the Bianchi identity. 


\section{References}

[1] E. Witten, Nucl. Phys. B 471 (1996) 135 arXiv:hep-th/9602070.

[2] P. Horava and E. Witten, Nucl. Phys. B 460 (1996) 506 arXiv:hep-th/9510209; B 475 (1996) 94 arXiv:hep-th/9603142.

[3] H. P. Nilles, M. Olechowski and M. Yamaguchi, Phys. Lett. B 415 (1997) 24 arXiv:hep-th/9707143; Nucl. Phys. B 530 (1998) 43 arXiv:hep-th/9801030.

[4] I. Antoniadis and M. Quirós, Phys. Lett. B 416 (1998) 327 arXiv:hep-th/9707208].

[5] A. Lukas, B. A. Ovrut and D. Waldram, Nucl. Phys. B 532 (1998) 43 arXiv:hep-th/9710208; Phys. Rev. D 57 (1998) 7529 arXiv:hep-th/9711197.

[6] P. Horava, Phys. Rev. D 54 (1996) 7561 arXiv:hep-th/9608019;

J. R. Ellis, Z. Lalak, S. Pokorski and W. Pokorski, Nucl. Phys. B 540 (1999) 149 arXiv:hep-ph/9805377;

Z. Lalak, S. Pokorski and S. Thomas, Nucl. Phys. B 549 (1999) 63 arXiv:hep-ph/9807503;

J. R. Ellis, Z. Lalak and W. Pokorski, Nucl. Phys. B 559 (1999) 71 arXiv:hep-th/9811133.

[7] E. Witten, Phys. Lett. B 155 (1985) 151.

[8] J.-P. Derendinger, L. E. Ibáñez and H. P. Nilles, Nucl. Phys. B 267 (1986) 365.

[9] M. J. Duff, J. T. Liu and R. Minasian, Nucl. Phys. B 452 (1995) 261 arXiv:hep-th/9506126.

[10] K. Becker, M. Becker and A. Strominger, Nucl. Phys. B 456 (1995) 130 arXiv:hep-th/9507158;

M. Bershadsky, C. Vafa and V. Sadov, Nucl. Phys. B 463 (1996) 420 arXiv:hep-th/9511222.

[11] E. Cremmer, B. Julia and J. Scherk, Phys. Lett. B 76 (1978) 409.

[12] G. W. Gibbons and P. K. Townsend, Phys. Rev. Lett. 71 (1993) 3754 arXiv:hep-th/9307049;

D. M. Kaplan and J. Michelson, Phys. Rev. D 53 (1996) 3474 arXiv:hep-th/9510053.

[13] J.-P. Derendinger and R. Sauser, Nucl. Phys. B 598 (2001) 87 arXiv:hep-th/0009054. 
[14] G. W. Moore, G. Peradze and N. Saulina, Nucl. Phys. B 607 (2001) 117 arXiv:hep-th/0012104.

[15] A. Lukas, B. A. Ovrut and D. Waldram, Phys. Rev. D 59 (1999) 106005 arXiv:hep-th/9808101.

[16] A. Lukas, B. A. Ovrut and D. Waldram, JHEP 9904 (1999) 009 arXiv:hep-th/9901017.

[17] A. Strominger, Phys. Lett. B 383 (1996) 44 arXiv:hep-th/9512059;

P. K. Townsend, Nucl. Phys. Proc. Suppl. 58 (1997) 163 arXiv:hep-th/9609217;

P. Brax and J. Mourad, Phys. Lett. B 416 (1998) 295 arXiv:hep-th/9707246;

Z. Lalak, A. Lukas and B. A. Ovrut, Phys. Lett. B 425 (1998) 59 arXiv:hep-th/9709214.

[18] E. Lima, B. A. Ovrut, J. Park and R. Reinbacher, Nucl. Phys. B 614 (2001) 117 arXiv:hep-th/0101049;

E. Lima, B. A. Ovrut and J. Park, Nucl. Phys. B 626 (2002) 113 arXiv:hep-th/0102046.

[19] G. Curio and A. Krause, Nucl. Phys. B 643 (2002) 131 arXiv:hep-th/0108220.

[20] E. I. Buchbinder and B. A. Ovrut, Phys. Rev. D 69 (2004) 086010 arXiv:hep-th/0310112;

E. I. Buchbinder, Phys. Rev. D 70 (2004) 066008 arXiv:hep-th/0406101.

[21] E. I. Buchbinder, Nucl. Phys. B 711 (2005) 314 arXiv:hep-th/0411062;

K. Becker, M. Becker and A. Krause, Nucl. Phys. B 715 (2005) 349 arXiv:hep-th/0501130.

[22] E. I. Buchbinder, Nucl. Phys. B 728 (2005) 207 arXiv:hep-th/0507164.

[23] J.-P. Derendinger and R. Sauser, Nucl. Phys. B 582 (2000) 231 arXiv:hep-th/0003078.

[24] M. B. Green and J. H. Schwarz, Phys. Lett. B 149 (1984) 117.

[25] A. Bilal, J.-P. Derendinger and R. Sauser, Nucl. Phys. B 576 (2000) 347 arXiv:hep-th/9912150.

[26] A. Bilal and S. Metzger, Nucl. Phys. B 675 (2003) 416 arXiv:hep-th/0307152. 
[27] P. K. Townsend, Phys. Lett. B 373 (1996) 68 arXiv:hep-th/9512062;

O. Aharony, Nucl. Phys. B 476 (1996) 470 arXiv:hep-th/9604103;

E. Bergshoeff, M. de Roo and T. Ortin, Phys. Lett. B 386 (1996) 85 arXiv:hep-th/9606118.

[28] E. Witten, Nucl. Phys. B 463 (1996) 383 arXiv:hep-th/9512219]; J. Geom. Phys. 22 (1997) 103 arXiv:hep-th/9610234.

[29] L. E. Ibáñez and H. P. Nilles, Phys. Lett. B 169 (1986) 354.

[30] S. Ferrara, J. Wess and B. Zumino, Phys. Lett. B 51 (1974) 239;

W. Siegel, Phys. Lett. B 85 (1979) 333.

[31] C. P. Burgess, J.-P. Derendinger, F. Quevedo and M. Quirós, Phys. Lett. B 348 (1995) 428 arXiv:hep-th/9501065; Annals Phys. 250 (1996) 193 arXiv:hep-th/9505171.

[32] S. Cecotti, S. Ferrara and M. Villasante, Int. J. Mod. Phys. A 2 (1987) 1839.

[33] T. Kugo and S. Uehara, Nucl. Phys. B 226 (1983) 49; B 222 (1983) 125.

[34] J.-P. Derendinger, S. Ferrara, C. Kounnas and F. Zwirner, Nucl. Phys. B 372 (1992) 145.

[35] J.-P. Derendinger, L. E. Ibáñez and H. P. Nilles, Phys. Lett. B 155 (1985) 65;

M. Dine, R. Rohm, N. Seiberg and E. Witten, Phys. Lett. B 156 (1985) 55.

[36] J. P. Derendinger, F. Quevedo and M. Quirós, Nucl. Phys. B 428 (1994) 282 arXiv:hep-th/9402007.

[37] G. Veneziano and S. Yankielowicz, Phys. Lett. B 113 (1982) 231.

[38] S. P. de Alwis, Phys. Lett. B 392 (1997) 332 arXiv:hep-th/9609211. 